\title{
Covariance Algebras in Field Theory and Statistical Mechanics
}

\author{
Sergio Doplicher*, Daniel Kastler** and Derek W. Robinson** \\ University of Aix-Marseille, France
}

Received March 1, 1966

\begin{abstract}
Starting from a $C^{*}$-algebra $\mathfrak{A}$ and a locally compact group $T$ of automorphisms of $\mathfrak{A}$ we construct a "covariance algebra" $\mathfrak{A}_{1}^{T}$ with the property that the corresponding $*$-representations are in one-to-one correspondence with covariant representations of $\mathfrak{A}$ i.e. $*$-representations of $\mathfrak{A}$ in which the automorphisms are continuously unitarily implemented. We further construct for relativistic field theory an algebra $\mathfrak{A}_{1}^{T}(V)$ yielding the $*$-representations of $\mathfrak{A}$ in which the space time translations have their spectrum contained in $V$. The problem of denumerable occurence of superselection sectors is formulated as a condition on the spectrum of $\mathfrak{A}_{1}^{T}(V)$. Finally we consider the covariance algebra $\mathfrak{A}_{1}^{T}$ built with space translations alone and show its relevance for the discussion of equilibrium states in statistical mechanics, namely we restore in this framework the equivalence of uniqueness of the vacuum, irreducibility and a weak clustering property.
\end{abstract}

\section{§ I. Introduction}

In recent formulations of both relativistic field theory and statistical mechanics emphasis is being placed upon the algebraic structure of these theories. The algebraic approach is based on the specification of a $C^{*}$. algebra of observables with structure properties related to space-time or space and its positive forms or (physical) states. It is customary however to work with special representations of the algebra i.e. special states so chosen that the space-(time) automorphisms are represented by unitary operators of the representation space. Thus consideration is restricted to a family of states transformed into each other by the space(time) automorphisms. Such a restriction is hoped to be a partial characterization of the superselection sectors of relativistic field theory and the equilibrium states of statistical mechanics. It is even believed that in the relativistic case the extra requirement of positivity of the energy might be essentially sufficient to completely characterize the superselection sectors whose denumerable occurence is one of the most fascinating general problems in field theory. In statistical mechanics extra specialization such as invariance and clustering properties should also be neces-

* On leave of absence from Istituto di Fisica „G. Marconi“ - Roma.

** Postal address: Physique Théorique, Faculté des Sciences, Place Victor Hugo - Marseille (France).

1 Commun. math. Phys., Vol. 3 
sary, and perhaps sufficient to characterize the equilibrium states ${ }^{1}$. In this paper we wish to demonstrate that such specialization can again be formulated in an algebraic setting.

The general idea is to build from the quasi-local algebra $\mathfrak{A}$ and the group of automorphisms $T$ an auxiliary algebra which we denote $\mathfrak{A}_{1}^{T}$ and call a "covariance algebra" because it has the property that its *-representations yield the representations of $\mathfrak{A}$ for which $T$ is unitarily implemented. In fact the representations of $\mathfrak{A}_{1}^{T}$ will be in a one-to-one correspondence with these "covariant representations" of $\mathfrak{A}$. The construction of $\mathfrak{A}_{1}^{T}$ is a natural generalization of the construction of the group algebra $L_{1}(T)$. Whilst $L_{1}(T)$ is a linearization of $T$ by functions $x \in T \rightarrow f_{x}$ from $T$ to the complex numbers behaving like integrals

$$
\int f_{x} U(x) d x
$$

$\mathfrak{A}_{1}^{T}$ is an algebra of functions $X: x \in T \rightarrow X_{x} \in \mathfrak{A}$ from $T$ to $\mathfrak{A}$ behaving like integrals

$$
\int \pi\left(X_{x}\right) U(x) d x
$$

where $\pi$ is a covariant representation of $\mathfrak{A}$ and $U$ the associated unitary representation of $T$. The theory of $L_{1}(T)$ is developed from the observation that the multiplication and adjunction of operators of the type (a) is in fact independent of the representation and can be stated algebraically. The same observation in the case of the operators (b) provides the basis for the theory of the *-algebra $\mathfrak{A}_{1}^{T}$ whose product and *-operation are the corresponding generalization of the usual convolution and *-operation of $L_{1}(T)$. Our general construction applies to any $C^{*}$-algebra $\mathfrak{A}$ and any locally compact group $T$ of automorphisms which acts upon $\mathfrak{A}$ in a strongly continuous manner.

In the case of relativistic theory we turn our attention to the group $T$ of space-time translations and show that the further restriction to representations with positive spectrum can also be formulated algebraically. This is achieved by considering the quotient $\mathfrak{A}_{1}^{T}(V)$ by a spectral ideal $\bar{J}$ which in heuristic terms contains the projectors on the unphysical energy-momentum spectrum. The spectrum of $\mathfrak{A}_{1}^{T}(V)$ is tightly connected with the part $\hat{\mathfrak{A}}_{p}$ of the spectrum $\hat{\mathfrak{A}}$ of $\mathfrak{A}$ which is determined by irreducible covariant representations with physical energy-momentum spectrum. As these representations are essentially the natural candidates for the description of superselection sectors the denumerable occurence of the latter is thus converted into a spectral property of $\mathfrak{A}_{1}^{T}(V)$ which thus provides a mathematical frame for a synthetic study of $\hat{\mathfrak{A}}_{p}$ based on locality.

1 R. HAAG, private communication. 
The covariance algebra which we believe to be of interest for statistical mechanics is obtained by taking $T$ to be the group of spacetranslations. Using general asymptotic properties of $\mathfrak{A}$ we then consider some properties of the invariant states. We introduce a new notion of clustering properties defined with the aid of mean values. Our main result is then the equivalence of weak clustering of the invariant state, irreducibility of $\mathfrak{A}_{1}^{T}$ and uniqueness of an invariant vector in Hilbert space. This equivalence and other subsidiary results are generalizations of properties which are well known in relativistic field theory.

Section II is devoted to the definition of the general covariance algebra $\mathscr{A}_{1}^{T}$ as a Banach $*$-algebra. In Section III we show that the *-representations of $\mathfrak{A}_{1}^{T}$ yield the covariant representations of $\mathfrak{A}$ in a one-to-one fashion. Section IV presents a proof of the $A^{*}$-character of $\mathfrak{A}_{1}^{T}$. Section $V$ is concerned with the algebra $\mathfrak{A}_{1}^{T}(V)$ for the relativistic case, Section VI with states of interest in statistical mechanics. Our conclusions are summarized at the ends of the latter two sections.

\section{$\S$ II. The algebra $\mathfrak{2}_{1}^{T}$}

In the first three sections of this paper we deal with the following general situation: we are given a $C^{*}$-algebra $\mathfrak{A}$ (separable or not) and an Abelian locally compact group $T$ acting on $\mathfrak{A}$ in the sense that

i) to each $x \in T$ corresponds a $*$-automorphism of the algebra $\mathfrak{A}$ i.e. a mapping $A \in \mathfrak{A} \rightarrow A(x) \in \mathfrak{A}$ such that

$$
\left\{\begin{aligned}
(\alpha A+\beta B)(x) & =\alpha A(x)+\beta B(x) \quad A, B \in \mathfrak{A} \\
(A B)(x) & =A(x) B(x) \quad \alpha, \beta \text { complex numbers } \\
A^{*}(x) & =A(x)^{*}
\end{aligned}\right.
$$

(we recall that the automorphism is automatically norm-preserving i.e.

$$
\|A(x)\|=\|A\|
$$

for all $A \in \mathfrak{A}$, where \|\| denotes the $C^{*}$-norm in $\mathfrak{A}$.)

ii) the correspondence i) is a representation of $T$ in the automorphism group of $\mathfrak{A}$ i.e. one has

$$
\left\{\begin{aligned}
{[A(x)](y) } & =A(x+y) \quad x, y \in T \\
A(0) & =A
\end{aligned}\right.
$$

iii) for each $A \in \mathfrak{A} A(x)$ is a continuous function of $x \in T$ in the norm topology of $\mathfrak{A}$ i.e. for each $x \in T$ and $\varepsilon>0$ there is a neighbourhood $V$ of zero in $T$ such that $h \in V$ implies

$$
\|A(x+h)-A(x)\| \leqq \varepsilon .
$$

In physical applications, $\mathfrak{A}$ will be the algebra of quasi-local observables of field theory [1] and $T$ will be the group of translations 
either in space and time or in space alone. In fact our theory could be developed for a non-Abelian locally compact group without other changes that some complications in writing.

Concerning assumption iii) we first point out that we could equivalently require continuity of the mapping $x \in T \rightarrow A(x) \in \mathfrak{A}$ in the weak topology of $\mathfrak{A}$ with respect to its dual $\mathfrak{A}^{*}$ i.e. continuity of the functions $x \rightarrow \varphi(A(x))$ for all $A \in \mathfrak{A}$ and all states $\varphi$ over $\mathfrak{A}$ (see [2], 10.2 Corollary and [6], 2.6.4).

In this latter form assumption iii) is more motivated on physical grounds. Further mathematical motivation is provided by the following

Proposition. If the $C^{*}$-algebra $\mathfrak{A}$ is separable iii) is implied by the existence of a faithful representation in which the group $T$ is continuously unitarily implemented.

Proof. For a separable $\mathfrak{A}$ iii) is equivalent with measurability of all functions $x \rightarrow \varphi(A(x))$ for all $A \in \mathfrak{A}$ and $\varphi \in \mathfrak{A}^{*}$ (see [2], Theorems 3.5.3 and 10.2.3.). Let us assume the existence of a faithful *-representation $\pi$ of $\mathfrak{A}$ along with a strongly continuous unitary representation $U$ of $T$ such that

$$
\pi(A(x))=U(x) \pi(A) U(x)^{-1}, \quad x \in T, A \in \mathcal{A} .
$$

We then have that the functions $x \rightarrow \varphi(A(x))$ are continuous for all $\varphi$ belonging to the linear hull $\mathscr{F}_{\pi}$ of vectorial forms in the representation $\pi$. Furthermore, since $\pi$ is faithful, it is known that $\mathscr{F}_{\pi}$ is dense in $\mathfrak{A}^{*}$ equipped with the weak topology with respect to $\mathfrak{A}$.

Now we recall that separability of $\mathfrak{A}$ implies the existence of a denumerable complete system of weak neighbourhoods of zero in the unit ball of $\mathfrak{A}^{*}$ viz. the

$$
V_{n}=\left\{\varphi \in \mathfrak{Q}^{*}\left|\operatorname{Sup}_{i \leqq n}\right| \varphi\left(A_{i}\right) \lambda \mid \leqq \frac{1}{n}\right\}, \quad n=1,2, \ldots
$$

where $A_{i}, i=1,2, \ldots$, is a dense sequence of elements of $\mathfrak{A}$. Let us then take a $\varphi \in \mathfrak{A}^{*}$ and prove that $x \rightarrow \varphi(A(x))$ is measurable. We select a sequence $\varphi_{n} \in \mathscr{F}_{\pi}$ such that $\left\|\varphi_{n}\right\|=\|\varphi\|$ and $\varphi-\varphi_{n} \in V_{n}$. By the density of the $A_{i}$ we then have that

$$
\varphi_{n}(A) \underset{n=\infty}{\longrightarrow} \varphi(A)
$$

for each $A \in \mathfrak{A}$. Therefore the function $x \rightarrow \varphi(A(x))$ is a pointwise limit of continuous functions $\varphi_{n}(A(x))$ and is thus measurable.

A discussion of the motivation of assumption iii) in the non-separable case is given in the Appendix.

We now give the following

Definition 1. We denote by $\mathfrak{A}_{1}^{T}$ the set of all measurable functions $X$ of $T$ to $\mathfrak{A}$ :

$$
X: x \in T \rightarrow X_{x} \in \mathfrak{A}
$$


defined up to a set of measure zero and absolutely integrable i.e. such that ${ }^{2}$

$$
\|X\|_{1}=\int\left\|X_{x}\right\| d x<\infty \text {. }
$$

More generally $\mathfrak{Q}_{p}^{T}$ will denote the set of measurable functions such that

$$
\|X\|_{p}^{p}=\int\left\|X_{x}\right\|^{p} d x<\infty \cdot T
$$

On the other hand we will denote by $\mathfrak{A}_{\mathscr{C}_{0}}^{T}$ and $\mathfrak{A}_{\mathscr{K}}^{T}$ the sets of norm-continuous functions $X \rightarrow X_{x}$ respectively vanishing at infinity and with compact support. We will set

$$
\|X\|_{\infty}=\operatorname{Sup}_{x \in T}\left\|X_{x}\right\|, \quad X \in \mathfrak{Q}_{\mathscr{C}_{0}}^{T} .
$$

As a consequence of these definitions $\mathfrak{A}_{1}^{T}$ is a Banach space under the norm \|\|$_{1}$ given by $(5)$ and $\mathfrak{A}_{\mathscr{K}}^{T}$ is a dense subset of $\mathfrak{A}_{1}^{T}$ (and analogously for $\mathfrak{A}_{p}^{T}$ see [3], Chap. IV $\S 3 \mathrm{n}^{\circ} 4$ and $\S 5 \mathrm{n}^{\circ} 6$ ). Our definition of measurability is that of [3], Chapt. IV $\S 5 \mathrm{n}^{\circ} 1$ Prop. 1, i.e. the function $X: x \rightarrow X_{x}$ is measurable whenever to each compact set $K \subset T$ and $\varepsilon>0$ there is a compact set $K^{\prime} \subset K$ on which $X$ is norm-continuous such that the measure $m\left(K^{\prime}-K\right)$ of $K^{\prime}-K$ is less than $\varepsilon$ (this entails measurability of the scalar function $x \rightarrow\left\|X_{x}\right\|$ so that (5) and (6) make sense).

Comment on our notation: we chose to write the value $X_{x}$ of the function $X \in \mathfrak{A}_{1}^{T}$ with $x$ as an index to avoid conflict with the established notation $A(x)$ for the automorphic image of $A \in \mathfrak{A}$ in the translation $x$. In order to avoid confusion between elements of $\mathfrak{A}_{1}^{T}$ and elements of $\mathfrak{A}$ we will consistently use symbols like $X, Y, Z$ for the first and $A, B, C$ or $X_{x}, Y_{u}$ etc. . . f for the latter (in order to stress this distinction one could use symbols like $X$., where the point stands for a dummy variable, for elements of $\mathfrak{A}_{1}^{T}$, but we do not find this necessary). With our notation the symbol $X_{x-u}(u)$ used below means the image in the translation $u \in T$ of the value $X_{x-u} \in \mathfrak{A}$ of $X \in \mathfrak{A}_{1}^{T}$ at the spot $x-u \in T$.

Lemma 1. Given any two $X, Y \in \mathfrak{A}_{1}^{T}$ the integral

$$
(X * Y)_{x}=\int X_{u} Y_{x-u}(u) d u
$$

exists for almost all $x \in T$ and defines an element $X * Y$ of $\mathfrak{U}_{1}^{T}$ such that

$$
\|X * Y\|_{1} \leqq\|X\|_{1} \cdot\|Y\|_{1} .
$$

Proof. For the integral (8) to exist it is sufficient that the integrand be measurable and of integrable norm. Now given any $\varepsilon>0$ and compact $K$ there is a compact $K^{\prime} \subset K$ on which $u \rightarrow X_{u}$ is continuous and such

${ }^{2}$ When we wish to consider everywhere defined, absolutely integrable functions $x \rightarrow X_{x}$ (rather than classes of functions defined up to negligible sets) we will speak about everywhere defined elements of $\mathfrak{A}_{1}^{T}$. The measure $d x$ is the Haar measure on $T$. 
that $m\left(K-K^{\prime}\right)<\frac{\varepsilon}{2}$. Again there exists a compact $K^{\prime \prime} \subset K^{\prime}$ on which $u \rightarrow Y_{x-u}$ is continuous and such that $m\left(K^{\prime}-K^{\prime \prime}\right)<\frac{\varepsilon}{2}$. Using (3) and the continuity of products in $\mathfrak{A}$ we have that the integrand of (8) is continuous on $K^{\prime \prime} \subset K^{\prime}$ such that $m\left(K-K^{\prime \prime}\right)<\varepsilon$, and therefore is measurable. On the other hand we have

$$
\int\left\|X_{u} Y_{x-u}(u)\right\| d u \leqq \int\left\|X_{u}\right\| \cdot\left\|Y_{x-u}\right\| d u,
$$

the last expression being the ordinary convolution product of the $L_{1}$ functions $u \rightarrow\left\|X_{u}\right\|$ and $u \rightarrow\left\|Y_{u}\right\|$ which is therefore integrable almost everywhere in $x$ and determines a $L_{1}$-function of $x$ of norm not exceeding $\|X\|_{1} \cdot\|Y\|_{1}$.

Lemma 2. Given $X \in \mathfrak{A}_{1}^{T}$ the definition

$$
\left(X^{*}\right)_{x}=\left\{X_{-x}(x)\right\}^{*}
$$

determines an element $X^{*}$ of $\mathfrak{A}_{1}^{T}$ of norm equal to that of $X$ :

$$
\left\|X^{*}\right\|_{1}=\|X\|_{1} \text {. }
$$

Using (3) $X^{*}$ is immediately seen to be measurable and we have

$$
\int\left\|X_{-x}(x)^{*}\right\| d x=\int\left\|X_{-x}\right\| d x=\|X\|_{1} .
$$

Theorem 1. Under the norm (5), the product (8) and the adjoint operation (10) $\mathfrak{Q}_{1}^{T}$ is a Banach *-algebra.

Proof. $\mathfrak{A}_{1}^{T}$ is already known to be a Banach space. The product (8) is obviously bilinear and the adjoint-operation obviously antilinear and such that $X^{* *}=X$ for all $X$.

Thus the only properties which remain to be shown are the following

$$
\begin{array}{cc}
(X * Y) * Z=X *(Y * Z) & X, Y, Z \in \mathfrak{A}_{1}^{T} \\
(X * Y)^{*}=Y^{*} * X^{*} & X, Y \in \mathfrak{A}_{1}^{T} .
\end{array}
$$

Both properties result from a straighforward calculation. We have, by definition

$$
\begin{aligned}
\{(X * Y) * Z\}_{y} & =\int d x(X * Y)_{x} Z_{y-x}(x) \\
& =\int d x\left\{\int d u X_{u} Y_{x-u}(u)\right\} Z_{y-x}(x) .
\end{aligned}
$$

On the other hand we have

$$
\begin{aligned}
\{X *(Y * Z)\}_{y} & =\int d u X_{u}(Y * Z)_{y-u}(u) \\
& =\int d u X_{u}\left\{\int d x Y_{v}(u) Z_{y-u-v}(u+v)\right\} \\
& =\int d u X_{u}\left\{\int d x Y_{x-u}(u) Z_{y-x}(x)\right\}
\end{aligned}
$$

where we used the strong continuity (3) of the translation $u$ to perform it under the integral sign. Now permuting multiplication in $\mathfrak{A}$ (which is strongly continuous) with integral signs both expressions are seen to be 
equal to

$$
\iint d x d u X_{u} Y_{x-u}(u) Z_{y-x}(x)
$$

(the use of FuBINI's theorem, see [3] Chapt. V $\$ 8 n^{\circ} 1$, Theorem 1 , is allowable since the integrand of (14) is absolutely integrable with respect to $d x d u$ for almost all $y$; measurability is readily verified and (14) is in modulus smaller than

$$
\iint d x d u\left\|X_{u}\right\| \cdot\left\|Y_{x-u}\right\| \cdot\left\|Z_{y-x}\right\|,
$$

which exists for almost all $y$ as a double convolution of scalar $L_{1}$-functions).

Property (13) results from a straightforward calculation where strong continuity of the *-operation and of the translation by $x$ is used for permuting this operation with integrals: one has

$$
\begin{aligned}
{\left[(X * Y)^{*}\right]_{x} } & =(X * Y)_{-x}(x)^{*}=\left\{\int X_{u} Y_{-x-u}(u) d u\right\}(x)^{*} \\
& =\int Y_{-x-u}(u+x)^{*} X_{u}(x)^{*} d v=\int Y_{-v}(v)^{*} X_{v-x}(x)^{*} d v \\
& =\left(Y^{*} * X^{*}\right)_{x} .
\end{aligned}
$$

Theorem 2. If $X$ and $Y$ are everywhere defined elements respectively of $\mathfrak{A}_{1}^{T}$ and $\mathfrak{A}_{1}^{T} \cap \mathfrak{A}_{\mathscr{C}_{0}}^{T}$ the integral (8) exists everywhere in $x$ and defines an element $X * Y$ of $\mathfrak{A}_{1}^{T} \cap \mathfrak{Q}_{\mathscr{C}_{0}}^{T}$.

$\mathfrak{A}_{1}^{T} \cap \mathfrak{U}_{\mathscr{C}_{0}}^{T}$ is a two-sided ${ }^{*}$-ideal of $\mathfrak{A}_{1}^{T}$ and one has

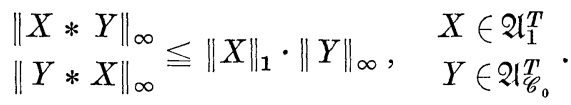

$\mathfrak{A}_{1}^{T} \cap \mathfrak{A}_{2}^{T}$ is also a two sided *-ideal of $\mathfrak{A}_{1}^{T}$ and one has

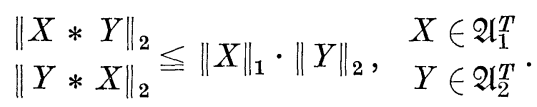

Proof. All sets considered being obviously invariant for the *-operation, it is enough to prove the left-ideal property. Take $X \in \mathfrak{A}_{1}^{T}$ and $Y \in \mathfrak{A}_{\mathscr{C}_{0}}^{T}$. The argument in the proof of Lemma 1 shows that the integral (8) exists for all $x \in T$. To prove the continuity of the function $X * Y$ at the spot $x$ we notice that $Y \in \mathfrak{A}_{\mathscr{C}_{0}}^{T}$ is uniformly continuous, so that, to each $\varepsilon>0$, we can choose a neighborhood $V$ of 0 in $T$ such that $h \in V$ implies

$$
\left\|Y_{x+h-u}-Y_{x-u}\right\|=\left\|Y_{x+h-u}(u)-Y_{x-u}(u)\right\|<\varepsilon
$$

for all $u \in T$. We then have

$\left\|(X * Y)_{x+h}-(X * Y)_{x}\right\| \leqq \int\left\|X_{u}\right\| \cdot\left\|Y_{x+h-u}(u)-Y_{x-u}(u)\right\| d u \leqq \varepsilon\|X\|_{1}$.

The fact that $X * Y$ tends towards zero at infinity follows from the majorization

$$
\left\|(X * Y)_{x}\right\| \leqq \int\left\|X_{u}\right\| \cdot\left\|Y_{x-u}\right\| d u
$$


where the second term is the ordinary convolution product of the functions $\left\{u \rightarrow\left\|X_{u}\right\|\right\} \in L_{1}(T)$ and $\left\{u \rightarrow\left\|Y_{u}\right\|\right\} \in \mathscr{C}_{0}(T)$ for which the result is well known. The same majorization settles the question of the first inequality (15) and also (using again a well-known property of ordinary convolution) of the first inequality (16), thereby proving that $\mathfrak{A}_{1}^{T} \cap \mathfrak{A}_{2}^{T}$ is a left-ideal. The other inequalities readily follow by use of the *-operation.

\section{$\S$ III. Representations of $\mathfrak{A}_{1}^{T}$ and covariant representations of $\mathfrak{A}$}

This section is devoted to the most important property of the algebra $\mathfrak{A}_{1}^{T}$ : the fact that there exists a one-to-one correspondence between covariant representations of $\mathfrak{A}$ and representations of $\mathfrak{Q}_{1}^{T}$ (see Theorem 3 below). This situation is a generalization of the situation encountered in the theory of the $L_{1}$-algebra of a locally compact group (which is obtained as a special case of the algebra $\mathfrak{A}_{1}^{T}$ when $\mathfrak{A}$ reduces to the complex number field trivially acted upon by $T$-commutativity of $T$ plays in fact no essential role as we mentioned above). Constructs analogous to the one we present here are to be found in the mathematical literature (DixMIER [4], Glimm [5] $\left.{ }^{3}\right)$.

The proof of Theorem 3 will be based on the consideration of operators $V(x)$ and $\varrho(A)$ on $\mathfrak{A}_{1}^{T}$ whose definition and relevant properties are gathered in Lemma 3. Lemma 4 establishes the existence of an approximate identity in $\mathfrak{A}_{1}^{T}-\mathrm{a}$ useful tool for questions pertaining to representations and positive forms.

Lemma 3. We associate with elements $x \in T$ and $A \in \mathfrak{A}$ the respective linear mappings $V(x)$ and $\varrho(A)$ on functions $x \rightarrow X_{x}$ from $T$ to $\mathfrak{A}$ defined as

$$
\left\{\begin{array}{lll}
V(x): X \rightarrow{ }^{x} X & \text { with } & \left({ }^{x} X\right)_{y}=Y_{y-x}(x) \\
\varrho(A): X \rightarrow{ }_{A} X & \text { with } & \left({ }_{A} X\right)_{y}=A X_{y}
\end{array}\right.
$$

These operators $V(x)$ and $\varrho(A)$ carry the respective spaces $\mathfrak{A}_{\mathscr{K}}^{T}, \mathfrak{H}_{\mathscr{C}_{0}}^{T}$ and $\mathfrak{A}_{1}^{T}$ (more generally $\mathfrak{I}_{p}^{(T)}$ into themselves with the following properties:

Properties of $V$ :

\begin{tabular}{|c|c|}
\hline$\left.V_{1}\right)$ & $\left\{\begin{aligned} V(x+y) & =V(x) V(y) \\
V(-x) & =V(x)^{-1}, \quad x, y \in T \\
V(0) & =\text { identity }\end{aligned}\right.$ \\
\hline$V_{J}$ & $\|V(x) X\|_{\infty}=\|X\|_{\infty}, \quad X \in \mathfrak{A}_{\mathscr{C}_{0}}^{T}$ \\
\hline$V_{1}$ & $\|V(x) X\|_{1}=\|X\|_{1}, \quad X \in \mathfrak{A}_{1}^{T}$ \\
\hline
\end{tabular}

${ }^{3}$ We are thankful to Dr. J. GLIMM for drawing our attention to his paper. 
$\left(V(x)\right.$ is more generally isometric with respect to the norm \|\|$_{p}$ in $\left.\mathfrak{A}_{p}^{T}\right)$ $\left.V_{4}\right)$

$\left.V_{5}\right)$

$$
\begin{aligned}
& \left\|V(x) X-V\left(x_{0}\right) X\right\|_{\infty} \underset{x=x_{0}}{\longrightarrow} 0, \quad X \in \mathfrak{A}_{\mathscr{C}_{0}}^{T} \\
& \left\|V(x) X-V\left(x_{0}\right) X\right\|_{1} \underset{x=x_{0}}{\longrightarrow} 0, \quad X \in \mathfrak{A}_{1}^{T}
\end{aligned}
$$

$\left(V(x)\right.$ is more generally continuous in $x$ for the norm \|\|$_{p}$ in $\left.\mathfrak{A}_{1}^{T}\right)$

$$
\left\{\begin{array}{l}
X^{*} * V(x) Y=\{V(-x) X\}^{*} * Y \\
\{V(x) X\}^{*} * V(x) Y=X^{*} * Y
\end{array}, \quad X, Y \in \mathfrak{A}_{1}^{T}\right.
$$

$\left.V_{7}\right)$

$$
\{V(x) X\} * Y=V(x)\{X * Y\}, \quad x \in T, \quad X, Y \in \mathfrak{A}_{1}^{T}
$$

Properties of $\varrho$ :

$$
\begin{gathered}
\varrho(A) \varrho(B)=\varrho(A B), \quad A, B \in \mathfrak{A} . \\
\|\varrho(A) X\|_{\infty} \leqq\|A\|\|X\|_{\infty}, \quad X \in \mathfrak{Q}_{\mathscr{C}_{0}}^{T} . \\
\|\varrho(A) X\|_{1} \leqq\|A\|\|X\|_{1}, \quad X \in \mathfrak{A}_{1}^{T} .
\end{gathered}
$$

(and analogous property in $\mathfrak{A}_{p}^{T}$ )

$\left.\varrho_{4}\right) \quad X^{*} * \varrho(A) Y=\left\{\varrho\left(A^{*}\right) X\right\}^{*} * Y, \quad A \in \mathfrak{U}, \quad X, Y \in \mathfrak{U}_{1}^{T}$.

$$
\{\varrho(A) X\} * \bullet \varrho(A) Y=X * * \varrho(A * A) Y
$$

$\left.\varrho_{5}\right)$ Let $A_{\lambda}, \lambda \in I$, be an approximate unit in $\mathfrak{A}$. One has

$$
\lim _{\lambda}\left\|\varrho\left(A_{\lambda}\right) X-X\right\|_{\infty}=0, \quad X \in \mathfrak{A}_{\mathscr{C}_{0}}^{T}
$$

and

$$
\lim _{\lambda}\left\|\varrho\left(A_{\lambda}\right) X-X\right\|_{1}=0, \quad X \in \mathfrak{U}_{1}^{T} .
$$

$\left.\varrho_{6}\right)$ The positive form on $\mathfrak{A}$ given by

$$
\psi(A)=\varphi\left(X^{*} * \varrho(A) X\right),
$$

where $\varphi$ is a positive form on $\mathfrak{U}_{1}^{T}$ and $X \in \mathfrak{U}_{1}^{T}$, has its norm equal to

$$
\|\psi\|=\varphi\left(X^{*} * X\right) \text {. }
$$

$\left.\varrho_{7}\right) \quad\{\varrho(A) X\} * Y=\varrho(A)\{X * Y\}, \quad A \in \mathfrak{A}, \quad X, Y \in \mathfrak{A}_{1}^{T}$.

Properties of $V$ and $\varrho$ :

$\left.V \varrho_{1}\right)$ For each $x \in T, A \in \mathfrak{A}$ one has

$\left.V \varrho_{2}\right)$ One has

$$
V(x) \varrho(A) V(x)^{-1}=\varrho(A(x)) .
$$

$$
X * Y=\int \varrho\left(X_{x}\right) V(x) Y d x \quad X, Y \in \mathfrak{A}_{1}^{T} .
$$

Notice that the properties contained in this lemma formally result from the fact that $\varrho(A) V(x)$ is equal to the left hand convolution times $A \delta_{x}$. This operation can be given a precise meaning by developing the theory of vector valued bounded measures on $T$ with values in $\mathfrak{A}$. We do not discuss this theory in the present paper, although the corresponding enlargement of the analytical apparatus is of interest for some questions (vector valued distributions may also be interesting e.g. for the description of the Hamiltonian). 
Proof of the lemma: The properties $V_{1}$ ) and $\varrho_{1}$ ) are obvious. If $X$ is strongly measurable, the same is true for ${ }^{x} X$ and ${ }_{A} X$ and since one has

and

$$
\left\|\left({ }^{x} X\right)_{y}\right\|=\left\|X_{y-x}\right\|
$$

$$
\left\|A X_{y}\right\| \leqq\|A\| \cdot\left\|X_{y}\right\|
$$

one sees that $V(x)$ and $\varrho(A)$ map the spaces $\mathfrak{A}_{\mathscr{b}_{0}}^{T}$, and $\mathfrak{A}_{1}^{T}$ (more generally $\left.\mathfrak{2}_{p}^{T}\right)$ into themselves with the properties $\left.V_{2}\right), V_{3}$ ) and $\left.\varrho_{2}\right), \varrho_{3}$ ).

To prove $V_{4}$ ) and $V_{5}$ ) we may take $x_{0}=0$ in view of $V_{1}$ ). Let us first take $X \in \mathfrak{A}_{\mathscr{K}}^{T}$ and consider

$$
\begin{aligned}
\left\|X_{y-x}(x)-X_{y}\right\| & \leqq\left\|X_{y-x}(x)-X_{y}(x)\right\|+\left\|X_{y}(x)-X_{y}\right\| \\
& \leqq\left\|X_{y-x}-X_{y}\right\|+\left\|X_{y}(x)-X_{y}\right\| .
\end{aligned}
$$

For each $y$ the differences $\left\|X_{y-x}-X_{y}\right\|$ and $\left\|X_{y}(x)-X_{y}\right\|$ can be made arbitrarily small by choosing $x$ small enough. Since those differences are both continuous functions in $y$ with compact support they can furthermore be made arbitrarily small uniformly in $y$. This shows that one has

$$
\|V(x) X-X\|_{\infty} \underset{x=0}{\longrightarrow} 0 \quad X \in \mathfrak{A}_{\mathscr{K}}^{T}
$$

and the same is true a fortiori for the norm \|\|$_{1}$. Properties $V_{4}$ ) and $V_{5}$ ) then immediately result by using the density of $\mathfrak{A}_{\mathscr{K}}^{T}$ respectively in $\mathfrak{A}_{\mathscr{\mathscr { C }}}^{T}$ for the norm \|\|$_{\infty}$ and in $\mathfrak{A}_{1}^{T}$ for the norm \|\|$_{1}$.

$\left.V_{6}\right), V_{7}$ ) and $\varrho_{4}$ ) are obtained by a straightforward calculation using the definition of the product and of the adjoint operation in $\mathfrak{A}_{1}^{T}$.

$\varrho_{4}$ ) shows that the form $\psi$ defined in $\varrho_{6}$ ) is a positive form on $\mathfrak{A}$. If $A_{\lambda}, \lambda \in I$, is an approximate identity in $\mathfrak{A}$ one then has (see [6] 2.1.5.(iv)):

$$
\|\psi\|=\lim _{\lambda} \psi\left(A_{\lambda}\right) .
$$

The proof of $\varrho_{6}$ ) is reduced to the proof of $\varrho_{5}$ ). For the latter, we first reduce it to the case of $X_{0} \in \mathfrak{A}_{\mathscr{K}}^{T}$ by writing, according to $\left.\varrho_{2}\right)$ and $\left.\varrho_{3}\right)$ :

$$
\begin{aligned}
\left\|\varrho\left(A_{\lambda}\right) X-X\right\|_{1} \leqq & \left\|\varrho\left(A_{\lambda}\right) X-\varrho\left(A_{\lambda}\right) X_{0}\right\|_{1}+ \\
& +\left\|\varrho\left(A_{\lambda}\right) X_{0}-X_{0}\right\|_{1}+\left\|X_{0}-X\right\|_{1} \\
\leqq & 2\left\|X-X_{0}\right\|_{1}+\left\|\varrho\left(A_{\lambda}\right) X_{0}-X_{0}\right\|_{\infty} .
\end{aligned}
$$

We then notice that one has for $X_{0} \in \mathfrak{A}_{\mathscr{K}}^{T}$

$$
\lim _{\lambda}\left\|\varrho\left(A_{\lambda}\right) X_{0}-X_{0}\right\|_{\infty}=0
$$

because $A_{\lambda} X_{0_{x}}-X_{0_{x}}$ tends to zero for each $x$, and therefore uniformly in $x$ by a compactness argument.

$\left.\varrho_{7}\right)$ is obvious from the definition of $\varrho$. 
$V \varrho_{1}$ ) is a straightforward consequence of the definition (18). Proof of $\left.V \varrho_{2}\right)$ : choose arbitrarily a compact $K\left(T\right.$ and $\varepsilon>0$. There exists $K^{\prime} \subset K$ on which $X$ is continuous such that $m\left(K-K^{\prime}\right)<\varepsilon$. Owing to $\left.V_{5}\right)$ and $\left.\varrho_{3}\right)$ the expression

$$
\varrho\left(X_{x}\right) V(x) Y
$$

is then continuous on $K^{\prime}$ and thus measurable. This expression is thus integrable since its norm does not exceed $\left\|X_{x}\right\| \cdot\|Y\|_{1}$ and the integral is of norm less than $\|X\|_{1} \cdot\|Y\|_{1}$. It is therefore enough to verify $V \varrho_{2}$ ) for $X, Y$ in the dense set $\mathfrak{2}_{\mathscr{K}}^{T}$ in which case the integral exists also in the \|\|$_{\infty}$ norm and we are free to interchange the integration with the process of taking the value at a point $u \in T$. We then have

$$
\begin{aligned}
\left(\int \varrho\left(X_{x}\right) V(x) Y d x\right)_{u} & =\int\left[\varrho\left(X_{x}\right) V(x) Y\right]_{u} d x=\int X_{x} Y_{u-x}(x) d x \\
& =(X * Y)_{u}
\end{aligned}
$$

q.e.d.

Lemma 4. Let $A_{\lambda} \in \mathfrak{Q}, \lambda \in I$, be an approximate unit for the $C^{*}$-algebra 2. Let on the other hand $J_{x}$ be the set of neighbourhoods of $x \in T$. We choose for each $\mu \in J_{x}$ a positive element $\varepsilon_{x, \mu} \in L_{1}(T)$ of unit $L_{1}$-norm with support within the neighbourhood $\mu$.

Denoting generally by $A f, A \in \mathfrak{A}, f \in L_{1}(T)$ the element of $\mathfrak{A}_{1}^{T}$ given by

$$
(A f)_{u}=f(u) A, \quad u \in T
$$

we then have, for each $X \in \mathfrak{A}_{1}^{T}$

$$
\lim _{\lambda, \mu}\left\|\left(A_{\lambda} \varepsilon_{x, \mu}\right) * X-V(x) X\right\|_{1} \rightarrow 0,
$$

where the limit is taken with respect to the product filter $I \times J_{x}$. In particular $A_{\lambda} \varepsilon_{0, \mu} \in \mathfrak{A}_{1}^{T},(\lambda, \mu) \in I \times J_{x}$, is an approximate unit for $\mathfrak{A}_{1}^{T}$ :

$$
\begin{gathered}
\left\|A_{\lambda} \varepsilon_{0, \mu}\right\|_{1}=1 \\
\lim _{\lambda, \mu}\left\|\left(A_{\lambda, \varepsilon_{0}, \mu}\right) * X-X\right\|_{1} \rightarrow 0, \quad X \in \mathfrak{Q}_{1}^{T} .
\end{gathered}
$$

Given a $B \in \mathfrak{A}$, we have also that

$$
\lim _{\lambda, \mu}\left\|\left(B A_{\lambda} \varepsilon_{x, \mu}\right) * X-\varrho(B) V(x) X\right\|_{1} \rightarrow 0, \quad X \in \mathfrak{A}_{1}^{T} .
$$

In particular $\varrho(B)$ can be defined by the limiting process

$$
\lim _{\lambda, \mu}\left\|\left(B A_{\lambda} \varepsilon_{0, \mu}\right) * X-\varrho(B) X\right\|_{1} \rightarrow 0, \quad X \in \mathfrak{A}_{1}^{T} .
$$

Proof: $A f \in \mathfrak{Q}_{1}^{T}$ as defined by (19) is obviously such that

$$
(A f) * X=\varrho(A)[f * X], \quad X \in \mathfrak{A}_{1}^{T},
$$

where $f * X \in \mathfrak{A}_{1}^{T}$ is (legitimately) defined as follows

$$
(f * X)_{y}=\int f(u) X_{y-u}(u) d u, \quad y \in T .
$$


This allows us to write

$$
\begin{aligned}
& \left\|\left(A_{\lambda} \varepsilon_{x, \mu}\right) * X-V(x) X\right\|_{1}=\left\|\varrho\left(A_{\lambda}\right)\left[\varepsilon_{x, \mu} * X\right]-V(x) X\right\|_{1} \leqq \\
& \quad \leqq \varrho\left(A_{\lambda}\right)\left[\varepsilon_{x, \mu} * X\right]-\varrho\left(A_{\lambda}\right) V(x) X\left\|_{1}+\right\| \varrho\left(A_{\lambda}\right) V(x) X-V(x) X \|_{1} .
\end{aligned}
$$

By $\varrho_{2}$ ) in Lemma 3 , the first term is majorized by

$$
\left\|\varepsilon_{x, \mu} * X-V(x) X\right\|_{1},
$$

whilst, by $\varrho_{4}$ ) in the same Lemma, the second term tends to zero with respect to $\lambda$. The proof of (20) thus reduces to showing that (24) tends to zero with respect to $\mu$. Now we have

$$
\begin{aligned}
\left\|\left\{\varepsilon_{x, \mu} * X-V(x) X\right\}_{y}\right\| & =\left\|\int \varepsilon_{x, \mu}(u)\left[X_{y-u}(u)-X_{y-x}(x)\right] d u\right\| \leqq \\
& \leqq \operatorname{Sup}_{u \in \mu}\left\|X_{y-u}(u)-X_{y-x}(x)\right\| .
\end{aligned}
$$

(24) is thus majorized by the expression

$$
\operatorname{Sup}_{u \in \mu}\|V(u) X-V(x) X\|_{1},
$$

where convergence to zero is assured by $V_{5}$ ) of Lemma 3 .

The proof of (22), (23) is similar and left to the reader.

The fact that we can choose the $\varepsilon_{0, \mu}$ continuous with compact support in $(21 \mathrm{~b})$ immediately gives the proof of the following corollary, which will serve us in the proof of the $A^{*}$-character of $\mathfrak{Q}_{1}^{T}$ given in the next section.

Corollary. If $X \in \mathfrak{Q}_{1}^{T}$ is such that $X * Z=0$ (or $Z * X=0$ ) for each $Z \in \mathfrak{A}_{\mathscr{S}}^{T}$, then $X=0$.

We now come to the main result of this section. We first give the

Definition 2. A covariant representation $(\pi, U)$ of $\mathfrak{A}$ is a pair of a *-representation $\pi$ of $\mathfrak{A}$ on a Hilbert space $\mathscr{H}$ and a strongly continuous unitary representation $U$ of $T$ on $\mathscr{H}$ such that one has

$$
\pi(A(x))=U(x) \pi(A) U(x)^{-1} \quad x \in T, A \in \mathfrak{A}
$$

$(\pi, U)$ is said to be essential if $\pi$ is essential i.e. does not contain the zero representation as a subrepresentation. The system $(\pi, U)$ is said to be cyclic with cyclic vector $\psi_{0}$ if the set of vectors of $\mathscr{H}$ obtained by applying to $\psi_{0}$ arbitrary products of operators $\pi\left(A_{i}\right), A_{i} \in \mathfrak{A}$, and $U\left(x_{j}\right), x_{j} \in T$, is a total set in $\mathscr{H}$ (i.e. generates linearly a dense set in $\mathscr{H})$.

Theorem 3. There is a one-to-one correspondence $(\pi, U) \leftrightarrow \hat{\pi}$ between essential covariant representations $(\pi, U)$ of $\mathfrak{A}$ and essential $*$-representations $\hat{\pi}$ of $\mathfrak{A}_{1}^{T} .(\pi, U) \rightarrow \hat{\pi}$ is given by

$$
\hat{\pi}(X) \psi=\int \pi\left(X_{x}\right) U(x) \psi d x \quad X \in \mathfrak{A}_{1}^{T}, \psi \in \mathscr{H} .
$$

$\hat{\pi} \rightarrow(\pi, U)$ is given by the equalities

$$
\begin{aligned}
\pi(A) \hat{\pi}(X) & =\hat{\pi}(\varrho(A) X) \\
U(x) \hat{\pi}(X) & =\hat{\pi}(V(x) X)
\end{aligned}
$$


(which determines $\pi(A)$ and $U(x)$ owing to the fact that $\hat{\pi} i$ s essential) or alternatively by

$\pi(A)=$ strong limit $\hat{\pi}\left(\varrho(A) E_{\lambda}\right)$

$U(x)=\underset{\lambda}{\operatorname{strong}} \underset{\lambda}{\operatorname{limit}} \hat{\tau}\left(V(x) E_{\lambda}\right), E_{\lambda}$ approximate identity in $\mathfrak{A}_{1}^{T}$.

The weak closure of $\hat{\pi}\left(\mathfrak{U}_{1}^{T}\right)$ is the same as the von Neumann algebra generated by $\pi(\mathfrak{U})$ and $U(T)$. $\hat{\pi}$ on the one side, the system $(\pi, U)$ on the other are simultaneously cyclic (with the same cyclic vector) or irreducible.

Proof. Let us start from the essential representation $\hat{\pi}$ of $\mathfrak{A}_{1}^{T}$ on the Hilbert space $\mathscr{H}$. Since the set $\hat{\pi}(X) \psi, X \in \mathfrak{U}_{1}^{T}, \psi \in \mathscr{H}$ is a total set in $\mathscr{H}$, the existence of the strong limits $\pi(A)$ and $V(x)$ of formulae $(28 \mathrm{a}, \mathrm{b})$ follows from

$$
\begin{aligned}
& \left\|\hat{\pi}\left(\varrho(A) E_{\lambda}\right) \hat{\pi}(X)-\hat{\pi}(\varrho(A) X)\right\| \leqq\|A\|\left\|E_{\lambda} * X-X\right\|_{1} \underset{\lambda}{\longrightarrow} 0 \\
& \left\|\hat{\pi}\left(V(x) E_{\lambda}\right) \hat{\pi}(X)-\hat{\pi}(V(x) X)\right\| \leqq\left\|E_{\lambda} * X-X\right\|_{1} \underset{\lambda}{\longrightarrow} 0
\end{aligned}
$$

where $X$ is an arbitrary element of $\mathfrak{A}_{1}^{T}$ and we have used $\left.\varrho_{7}\right), \varrho_{3}$ ) and $V_{7}$ ), $V_{3}$ ) of Lemma $3 . \pi$ and $U$ are respectively a $*$-representation of $\mathfrak{A}$ and a unitary representation of $T$ owing to $\left.\varrho_{1}\right), \varrho_{4}$ ) and $\left.V_{1}\right), V_{6}$ ) using again the fact that $\hat{\pi}$ is essential. The strong continuity of $U(x)$ in $x$ will result from the continuity of all matrix elements between vectors of a total set, we have namely

$$
\begin{aligned}
\left|\left(\hat{\pi}\left(X^{\prime}\right) \psi^{\prime}\left|U(x)-U\left(x_{0}\right)\right| \hat{\pi}(X) \psi\right)\right| & \\
= & \left(\psi^{\prime}\left|\hat{\pi}\left(X^{\prime *} *\left\{\left[V(x)-V\left(x_{0}\right)\right] X\right\} \mid \psi\right)\right| \leqq\right. \\
& \leqq\left\|\psi^{\prime}\right\| \cdot\|\psi\| \cdot\left\|X^{\prime}\right\|_{1}\left\|V(x) X-V\left(x_{0}\right) X\right\|_{1}
\end{aligned}
$$

for arbitrary $X, X^{\prime} \in \mathfrak{A}_{1}^{T}$ and $\psi, \psi^{\prime} \in \mathscr{H}$, the last expression tending to zero for $x \rightarrow x_{0}$ by $V_{5}$ ). The intertwining property (25) needs only be checked on the total set of the $\hat{\pi}(X) \psi$ where it follows from $(27 \mathrm{a}, \mathrm{b})$ and $\left.V \varrho_{1}\right)$. The fact that $\pi$ is essential results from the property

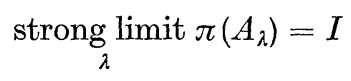

for any approximate identity $A_{\lambda}$ of $\mathfrak{A}$. (29) follows from

$$
\begin{aligned}
\left\|\pi\left(A_{\lambda}\right) \hat{\pi}(X) \psi-\hat{\pi}(X) \psi\right\|= & \left\|\pi\left(\varrho\left(A_{\lambda}\right) X-X\right) \psi\right\| \leqq \\
& \leqq\left\|\varrho\left(A_{\lambda}\right) X-X\right\|_{1}\|\psi\| \underset{\lambda}{\longrightarrow} 0
\end{aligned}
$$

for any $X \in \mathfrak{A}_{1}^{T}$ and $\psi \in \mathscr{H}\left(\right.$ we used $\left.\left.\varrho_{5}\right)\right)$.

Let us now start from a covariant representation $(\pi, U)$ of $\mathfrak{A}$. Given $X \in \mathfrak{A}_{1}^{T}$ we define $\hat{\tau}(X)$ by $(26)$, where the expression under the integral sign is continuous (by the strong continuity (3) of the translation automorphisms) and of norm $\leqq\left\|X_{u}\right\|$. The fact that $\hat{\tau}$ is a $*$-representation follows from an easy calculation using the Fubini theorem ([3] loc. cit.) and 
the permutability of integrals and continuous linear transformations. We have

and

$$
\begin{aligned}
\hat{\pi}(X) \hat{\pi}(Y) & =\int \pi\left(X_{u}\right) U(u) d u \int \pi\left(Y_{v}\right) U(v) d v \\
& =\int d u \int \pi\left(X_{u} Y_{v-u}(u)\right) U(v) d v \\
& =\int d v \pi\left\{\int X_{u} Y_{v-u} d u\right\} U(v) d v=\hat{\pi}(X * Y)
\end{aligned}
$$

$$
\begin{aligned}
\hat{\pi}\left(X^{*}\right) & =\int \pi\left(\left(X^{*}\right)_{u}\right) U(u) d u=\int \pi\left(X_{-u}(u)^{*}\right) U(u) d u \\
& =\int U(u) \pi\left(X_{-u}\right)^{*} d u=\hat{\pi}(X)^{*} .
\end{aligned}
$$

The representation $\hat{\pi}$ is essential: let namely $\Psi \in \mathscr{H}$ be such that $\hat{\pi}(X) \Psi=0$ for all $X \in \mathfrak{Q}_{1}^{T}$. Using the notations of Lemma 4 we have for all $A \in \mathfrak{A}$

$$
0=\lim _{\mu} \hat{\pi}\left(A \varepsilon_{0, \mu}\right) \Psi=\pi(A) \lim _{\mu} \int \varepsilon_{0, \mu}(u) U(u) d u \cdot \Psi=\pi(A) \Psi
$$

whence $\Psi=0$ due to the essential character of $\pi$.

We now prove that the correspondances $\hat{\pi} \stackrel{\Phi_{1}}{\longrightarrow}(\pi, U)$ and $(\pi, U) \stackrel{\Phi_{2}}{\longrightarrow} \hat{\pi}$ respectively defined by $(26)$ and $(27 \mathrm{a}, \mathrm{b})$ are the inverse of each other. Let $Y$ and $\Psi$ be arbitrary respectively in $\mathfrak{A}_{1}^{T}$ and $\mathscr{H} \cdot\left(\pi^{\prime}, U^{\prime}\right)$ $=\Phi_{1} \circ \Phi_{2}\{(\pi, U)\}$ is such that, for all $A \in \mathcal{A}$ and $x \in T$

and

$$
\begin{aligned}
\pi^{\prime}(A) \hat{\pi}(Y) \Psi & =\hat{\pi}(\varrho(A) Y) \Psi=\int \pi\left(A Y_{u}\right) U(u) \Psi d u \\
& =\pi(A) \int \pi\left(Y_{u}\right) U(u) \Psi d u=\pi(A) \hat{\pi}(Y) \Psi
\end{aligned}
$$

$$
\begin{aligned}
U^{\prime}(x) \hat{\pi}(Y) \Psi & =\hat{\pi}(V(x) Y) \Psi=\int \pi\left(Y_{u-x}(x)\right) U(u) \Psi d u \\
& =\int U(x) \pi\left(Y_{u-x}\right) U(u-x) \Psi d u=U(x) \hat{\pi}(Y) \Psi
\end{aligned}
$$

where $\hat{\pi}=\Phi_{2}\{(\pi, U)\}$ is cyclic so that $\pi^{\prime}=\pi$ and $U^{\prime}=U$. Conversely $\hat{\pi}^{\prime}=\Phi_{2} \circ \Phi_{1}\{\hat{\pi}\}$ is such that, for all $X \in \mathfrak{A}_{1}^{T}$

$$
\begin{aligned}
\hat{\pi}^{\prime}(X) \hat{\pi}(Y) \Psi & =\int \pi\left(X_{u}\right) U(u) \hat{\pi}(Y) \Psi d u=\int \hat{\pi}\left(\varrho\left(X_{u}\right) V(u) Y\right) \Psi d u \\
& =\hat{\pi}(X * Y) \Psi=\hat{\pi}(X) \hat{\pi}(Y) \Psi
\end{aligned}
$$

whence $\hat{\pi}^{\prime}=\hat{\pi}$ (we had $(\pi, U)=\Phi_{1}\{\hat{\pi}\}$ and used $V \varrho_{2}$ ) of Lemma 3 .

Let now $B$ be a bounded linear operator on $\mathscr{H}$. If $B \in \pi(\mathfrak{A})^{\prime} \cap U(T)^{\prime}$ (26) shows that $B \in \hat{\pi}\left(\mathfrak{A}_{1}^{T}\right)^{\prime}$. It follows from $(28 \mathrm{a}, \mathrm{b})$ that the converse is also true. $\hat{\pi}(\mathfrak{Q})$ on one side, $\pi(\mathfrak{R})$ and $U(T)$ on the other generate therefore the same von Neumann algebra. The last part of the theorem follows from the observation that cyclicity of a vector $\Psi \in \mathscr{H}$ for a system of operators $\mathfrak{M}$ (defined as the fact that $\mathfrak{M} \Psi$ is a total set in $\mathscr{H}$ ) is equivalent with cyclicity of $\Psi$ for the von Neumann algebra generated by $\mathfrak{M}$.

We will not discuss fully in this paper the relationship between positive linear forms on $\mathfrak{A}$ and positive linear forms on $\mathfrak{A}_{1}^{T}$. We merely give the 
Theorem 4. Let $\hat{\Phi}$ be a positive linear form an $\mathfrak{A}_{1}^{T}$ and $E_{\lambda}$ an approximate unit of $\mathfrak{A}_{1}^{T}$. The limit

$$
\Phi(A)=\lim _{\lambda} \hat{\Phi}\left(\varrho(A) E_{\lambda}\right)
$$

exists for all $A \in \mathfrak{A}$ and defines a positive form $\Phi$ on $\mathfrak{A}$. If $\hat{\pi}$ is the cyclic representation of $\mathfrak{A}_{1}^{T}$ generated by $\hat{\Phi}, \Psi$ the corresponding cyclic vector and $\pi$ the representation of $\mathfrak{A}$ associated to $\hat{\pi}$ by Theorem 3 one has

$$
\Phi(A)=(\Psi|\pi(A)| \Psi) .
$$

In other terms the representation of $\mathfrak{A}$ generated by $\Phi$ is the cyclic component of $\Psi$ in the representation $\pi$ of $\mathfrak{A}$.

Proof: We have by (28a)

$$
\begin{aligned}
\lim _{\lambda} \hat{\Phi}\left(\varrho(A) E_{\lambda}\right) & =\lim _{\lambda}\left(\Psi\left|\hat{\pi}\left(\varrho(A) E_{\lambda}\right)\right| \Psi\right) \\
& =(\Psi|\pi(A)| \Psi)
\end{aligned}
$$

\section{$\S$ IV. The $A^{*}$-character of $\mathfrak{A}_{1}^{T}$}

We introduce this section by stating properties of $\mathfrak{A}_{1}^{T}$ which follow from the fact that it is a Banach $*$-algebra with an approximate unit. Firstly [7] every positive form on $\mathfrak{A}_{1}^{T}$ will automatically be continuous. Secondly (see [6], 2.7) given $X \in \mathfrak{A}_{1}^{T}$ we have the equality of the four different definitions

$$
\left\{\begin{aligned}
\|X\| & =\operatorname{Sup}_{\text {all } * \text {-representations } \hat{\imath} \text { of } \mathfrak{2}_{1}^{T}}\{\|\hat{\pi}(X)\|\} \\
& =\operatorname{Sup}_{\text {all irreducible representations } \hat{\imath}}\{\|\hat{\pi}(X)\|\} \\
& =\operatorname{Sup}_{\text {all positive forms } \varphi \text { of unit norm on } \mathfrak{2}_{1}^{T}}\left\{\varphi\left(X^{*} * X\right)^{1 / 2}\right\} \\
& =\operatorname{Sup}_{\text {all pure normalized states } \varphi}\left\{\varphi\left(X^{*} * X\right)^{1 / 2}\right\} .
\end{aligned}\right.
$$

Moreover one defines in this way a pseudo-norm \|\| on $\mathfrak{A}_{1}^{T}$ which satisfies the "Gelfand Neumark identity":

$$
\left\|X^{*} * X\right\|=\|X\|^{2}, \quad X \in \mathfrak{A}_{1}^{T} .
$$

We will now prove the following Theorem - a further step towards showing that our theory is a generalization of the $L_{1}$-theory of a locally compact group.

Theorem 5. $\mathfrak{A}_{1}^{T}$ is an $A^{*}$-algebra i.e. (32) defines a norm on $\mathfrak{A}_{1}^{T}$. Under this norm (the minimal regular norm, see [8] §18), $\mathfrak{A}_{1}^{T}$ is according to (32) an incomplete $C^{*}$-algebra. Its completion (the enveloping $C^{*}$-algebra of $\mathfrak{A}_{1}^{T}$ ) will be denoted $\overline{\mathfrak{A}}_{1}^{T}$. 
Proof of the theorem: we have to show that $\|X\|=0$ implies $X=0$. To this end we display a set $\mathfrak{S}$ of positive linear forms on $\mathfrak{A}_{1}^{T}$ such that $f\left(X^{*} * X\right)=0$ for all $f \in \mathscr{\subseteq}$ implies $X=0$. The set $\subseteq$ is provided by the following

Lemma 5. Let $\varphi$ be a positive linear form on $\mathfrak{A}$ and $Z$ be an element of $\mathfrak{A}_{1}^{T} \cap \mathfrak{A}_{\mathscr{C}_{0}}^{T}$. Let us set, for each $X \in \mathfrak{R}_{1}^{T}$

$$
\varphi_{Z}(X)=\varphi\left\{\left(Z^{*} * X * Z\right)_{0}\right\} .
$$

We get in this way a positive linear form on $\mathfrak{2}_{1}^{T}$ such that

$$
\varphi_{Z}\left(X^{*} * X\right)=\int \varphi\left\{\left[(X * Z)_{-u}^{*}(X * Z)_{-u}\right](u)\right\} d u .
$$

The proof of this lemma is straightforward: given $Y, Z \in \mathfrak{A}_{1}^{T} \cap \mathfrak{A}_{\mathscr{C}_{\mathrm{G}}}^{T}$ one has $Z^{*} * Y \in \mathfrak{A}_{1}^{T} \cap \mathfrak{A}_{\mathscr{C}_{0}}^{T}$ and

$$
\left(Z^{*} * Y\right)_{0}=\int\left(Z_{-u}\right) *(u) Y_{-u}(u) d u=\int\left\{\left(Y_{-u}\right) * Z_{-u}\right\}(u) d u .
$$

As the positive linear form $\varphi$ is continuous one has

$$
\varphi\left\{\left(Z^{*} * Y\right)_{0}\right\}=\int \varphi\left\{\left(Z_{-u}\right) *(u) Y_{-u}(u)\right\} d u .
$$

In particular $\varphi\left\{\left(Y^{*} * Y\right)_{0}\right\} \geqq 0$. (33) is obtained by setting $Y=X * Z$ which is an element of $\mathfrak{A}_{1}^{T} \cap \mathfrak{A}_{\mathscr{C}_{0}}^{T}$ by Theorem 2, (34) being then equal tc $\varphi\left\{\left(Y^{*} * Y\right)_{0}\right\} \geqq 0$.

We now resume the proof of the theorem. We take for $\mathfrak{E}$ the set of all $\varphi_{Z}$ where $\varphi$ runs through the positive forms on $\mathfrak{A}$ and $Z$ runs through. $\mathfrak{A}_{1}^{T} \cap \mathfrak{A}_{\mathscr{C}_{0}}^{T}$. The integrand in the right hand side of (34) being continuous $\varphi_{Z}\left(X^{*} * X\right)=0$ implies

$$
\varphi\left\{\left[(X * Z)_{-u}^{*}(X * Z)_{-u}\right](u)\right\}=0
$$

for all $u \in T$. $\varphi$ being arbitrary one has also

$$
\varphi\left\{\left[(X * Z)_{-u}^{*}(X * Z)_{-u}\right]\right\}=0
$$

for all $\varphi$ and $u$, whence, since $\mathfrak{A}$ is a $C^{*}$-algebra, $(X * Z)_{u}=0$ for all $u$ $X$ is thus such that $X * Z=0$ for all $Z \in \mathfrak{A}_{1}^{T} \cap \mathfrak{V}_{\mathscr{C}_{0}}^{T}$. Since we can choost an approximate unit of $\mathfrak{U}_{1}^{T}$ within $\mathfrak{U}_{1}^{T} * \mathfrak{A}_{\mathscr{C}}^{T}$ we then have $X=0$ by $(22$ of Lemma 4.

\section{$\S \mathrm{V}$. The algebra $\mathfrak{A}_{1}^{T}(\boldsymbol{V})$}

In this section $\mathfrak{A}$ will be the quasi-local algebra of a relativistic fielc theory and $T$ the group of space time translations. We denote by $V$; closed subset of the energy-momentum space $\hat{T}$ (in practice $V$ will be (i closed subset of) the closed forward light cone $\bar{V}_{+}$) and focus our atten tion on the covariant representations of $\mathfrak{A}$ with energy momentun spectrum lying within $V$ (which we call the $V$-representations). In wha follows we construct an $A^{*}$-algebra $\mathfrak{A}_{1}^{T}(V)$ whose continuous $*$-represen tations are in one-to-one correspondence with the $V$-representations Let us first give the 
Definition 3. We denote by $J(V)$ the (two-sided) *-ideal of $\mathfrak{A}_{1}^{T}$ generated by the elements Af (see definition in Lemma 4), where A runs through $\mathfrak{A}$ and $f$ runs through all $L_{1}$-functions on $T$ such that the support of their Fourier-transform $\hat{f}$ does not intersect $V$. Furthermore $\bar{J}(V)$ denotes the norm-closure of $J(V)$ in $\overline{\mathfrak{A}}_{1}^{T}$ (i.e. the smallest closed $*$-ideal of $\overline{\mathfrak{A}}_{1}^{T}$ containing $J)$. We then call $\mathfrak{A}_{1}^{T}(V)$ the quotient of $\mathfrak{A}_{1}^{T}$ through its two-sided $*$-ideal $\mathfrak{A}_{1}^{T} \cap \bar{J}(V) . \overline{\mathfrak{A}}_{1}^{T}(V)$ will denote the quotient of $\mathfrak{A}_{1}^{T}$ through $\bar{J}(V)$ :

$$
\begin{gathered}
\mathfrak{A}_{1}^{T}(V)=\mathfrak{A}_{1}^{T} / \mathfrak{A}_{1}^{T} \cap \bar{J}(V) \\
\overline{\mathfrak{A}_{1}^{T}}(V)=\overline{\mathfrak{A}_{1}^{T}} / \bar{J}(V) .
\end{gathered}
$$

$\mathfrak{A}_{1}^{T} \cap \bar{J}(V)$ is the closure of $J(V) \subset \mathfrak{A}_{1}^{T}$ in the topology of $\mathfrak{A}_{1}^{T}$ determined by the $C^{*}$-norm \|\| . Since this topology is weaker than the \|\|$_{1}$-topology, $\mathfrak{A}_{1}^{T} \cap \bar{J}(V)$ is also closed in the \|\|$_{1}$-topology. Let $A$ be an arbitrary element of $\mathfrak{A}_{1}^{T}$, call $\hat{A} \in \mathfrak{A}_{1}^{T}(V)$ the corresponding class modulo $\bar{J}(V)$. If we define

and

$$
\|\hat{A}\|_{1}=\operatorname{Inf}\left\{\|A+K\|_{1} \mid K \in \mathfrak{A}_{1}^{T} \cap \bar{J}(V)\right\}
$$

$$
\|A\|=\operatorname{Inf}\left\{\|A+K\| \mid K \in \mathfrak{A}_{1}^{T} \cap \bar{J}(V)\right\}
$$

we get two norms on $\mathfrak{A}_{1}^{T}(V)$ for both of which it is a normed $*$-algebra. Under the \|\|$_{1}$-norm $\mathfrak{A}_{1}^{T}(V)$ is a Banach $*$-algebra, under the \|\| -norm it is an incomplete $C^{*}$-algebra whose completion coincides with $\overline{\mathfrak{A}}_{1}^{T}(V)$.

The following four kinds of objects are obviously mutually in a one-to-one correspondence:

- the (continuous) *-representations of $\mathfrak{Q}_{1}^{T}(V)$

- the (continuous) *-representations of $\mathfrak{U}_{1}^{T}$ vanishing on $\bar{J}(V) \cap \mathfrak{A}_{1}^{T}$

- the (continuous) *-representations of $\overline{\mathfrak{A}}_{1}^{T}(V)$

- the (continuous) *-representations of $\overline{\mathfrak{A}}_{1}^{T}$ vanishing on $\bar{J}(V)$

(note that $\bar{J}(V)$ is the closure of $\bar{J}(V) \cap \mathfrak{Q}_{1}^{T}$ in $\overline{\mathfrak{Q}}_{1}^{T}$ ). This shows that the \|\| -norm $(38)$ on $\mathfrak{A}_{1}^{T}(V)$ is the minimal regular norm.

On the other hand Theorem 3 establishes a one-to-one mapping between $*$-representations $\hat{\pi}$ of $\mathfrak{R}_{1}^{T}$ and covariant representations $(\pi, U)$ of $\mathcal{A}$. Let $f$ be an arbitrary $L_{1}$-function on $T$ and $\hat{f}$ its Fourier transform. For each $A \in \mathfrak{A}$

$$
\hat{\pi}(A f)=\pi(A) \int f(x) U(x) d x=\pi(A) \int \hat{f}(p) d E(p),
$$

where $d E(p)$ is the spectral measure associated with the translations:

$$
U(x)=\int e^{i p x} d E(p), \quad x \in T .
$$

If $\hat{\pi}\left(\bar{J}(V) \cap \mathfrak{A}_{1}^{T}\right)=0$, (39) vanishes whenever the support of $\hat{f}$ does not intersect $V$. Due to the arbitrariness of $A \in \mathfrak{A}$ and the cyclicity of $\pi$ we then have that

$$
\int \hat{f}(p) d E(p)=0,
$$

2 Commun. math. Phys., Vol. 3 
from which we conclude that the spectral measure $d E(p)$ has its support within $V$. Conversely if this is the case (41) and (40) vanish whenever supp $\hat{f} \cap V=\phi$ whence $\hat{\pi}\left(\bar{J}(V) \cap \mathfrak{A}_{1}^{T}\right)=0$. We thus have obtained (i) of the following

Theorem 6. (i) There is a one-to-one correspondence between covariant representations $(\pi, U)$ of $\mathfrak{A}$ with spectrum included in $V$ and continuous *-representations $\tilde{\pi}$ of $\mathfrak{A}_{1}^{T}(V)$ (or, for that matter, *-representations of $\left.\overline{\mathfrak{A}}_{1}^{T}(V)\right) . \tilde{\pi}$ is such that $\hat{\pi}=\tilde{\pi} \circ i$ where $\hat{\pi}$ is the representation of $\mathfrak{A}_{1}^{T}$ associated by Theorem 3 with the system $(\pi, U)$ and $i$ the canonical mapping from $\mathfrak{A}_{1}^{T}$ onto $\mathfrak{A}_{1}^{T}(V)$.

(ii) If $V \subset \bar{V}_{+}, \tilde{\pi}$ is irreducible if and only if $\pi$ is irreducible.

(iii) Let $\tilde{\pi}_{\alpha}$ and $\tilde{\pi}_{\beta}$ be two irreducible representations of $\mathfrak{Q}_{1}^{T}(V), V \subset \bar{V}_{+}$, $\left(\pi_{\alpha}, U_{\alpha}\right)$ and $\left(\pi_{\beta}, U_{\beta}\right)$ the corresponding covariant representations of $\mathfrak{A}$. Suppose furthermore that $\pi_{1}$ and $\pi_{2}$ are unitarily equivalent with intertwining operator $W$ :

$$
\pi_{\alpha}(A)=W \pi_{\beta}(A) W^{-1}, \quad A \in \mathfrak{A} .
$$

Then there exists a character $p$ of $T$ such that

$$
U_{\alpha}(x)=e^{i p x} W U_{\beta}(x) W^{-1}, \quad x \in T .
$$

(iv) The spectrum $\widehat{\mathfrak{A}_{1}^{T}\left(\widehat{V}_{+}\right)}$of $\overline{\mathfrak{A}}_{1}^{T}\left(\bar{V}_{+}\right)$is the set-theoretic product $\hat{\mathfrak{A}}_{p} \times{\overline{V_{+}}}^{4}$.

For the proof of (ii) and (iii) we shall use a result of Borchers ([9] or $[10])$ according to which, in a covariant representation $(\pi, U)$ of $\mathfrak{A}$ with positive spectrum, the operators $U(x), x \in T$, can be written as

$$
U(x)=U_{1}(x) U_{2}(x)
$$

where $U_{1}$ and $U_{2}$ are two-strongly continuous representations of $T$ with positive spectra such that

$$
\begin{aligned}
& U_{1}(x) \in \pi(\mathfrak{Q})^{\prime \prime} \\
& U_{2}(x) \in \pi(\mathfrak{A})^{\prime}, \quad x \in T .
\end{aligned}
$$

Let us consider a representation $\tilde{\pi}$ of $\mathfrak{A}_{1}^{T}(V)$ and the associated covariant representation $(\pi, U)$ of $\mathfrak{A}$. We have, by (44) and (45):

$$
U_{2}(T) \subset \pi(\mathfrak{A})^{\prime}=\left\{\pi(\mathfrak{A}) \cup U_{1}(T)\right\}^{\prime} .
$$

$T$ being Abelian, this implies

$$
U_{2}(T) \subset\left\{\pi(\mathfrak{Q}) \cup U_{1}(T) \cup U_{2}(T)\right\}^{\prime}=\{\pi(\mathfrak{A}) \cup U(T)\}^{\prime} .
$$

4 We recall that $\hat{\mathscr{A}}_{p}$ denotes the subset of $\hat{\mathfrak{U}}$ containing covariant irreducible representations with spectrum included in $\bar{V}_{+}$. 
In the special case of irreducibility of the representation $\tilde{\pi}$ (or equivalently of the system $(\pi, U)),\{\pi(\mathfrak{Q}) \cup U(T)\}^{\prime}$ reduces to the multiples of the identity, so that

$$
U_{2}(x)=e^{i p x} \cdot I, \quad x \in T
$$

for some $p \in \hat{T}$. Accordingly one has

$$
\pi(\mathfrak{R})^{\prime}=\left\{\pi(\mathfrak{R}) \cup U_{1}(T)\right\}^{\prime}=\{\pi(\mathfrak{R}) \cup U(T)\}^{\prime}
$$

and $\pi$ is irreducible. Conversely irreducibility of $\pi$ obviously implies irreducibility of $\tilde{\pi}$, which completes the proof of (ii).

To prove (iii) we write equation (42) for both $A$ and $A(x)$, thus getting that

$$
U_{\alpha}(x)^{-1} W U_{\beta}(x) W^{-1} \in \pi_{\alpha}(\mathfrak{A})^{\prime}
$$

so that, by (ii), one has

for some $p \in \hat{T}$.

$$
W U_{\beta}(x) W^{-1}=e^{i p x} U_{\alpha}(x)
$$

(iii) shows that the correspondence $\tilde{\pi} \in \widehat{\mathfrak{Q}_{1}^{T}}\left(\widehat{V_{+}}\right) \rightarrow \pi \in \hat{\mathfrak{Q}}_{p}$ is not oneto-one (we are now considering unitary equivalence classes of representations rather than the representations themselves). The different $\tilde{\pi}=(\pi, U)$ associated with a given irreducible $\pi \in \hat{\mathfrak{A}}_{p}$ are all obtained from one of them, say $\left(\pi, U_{0}\right)$ by multiplying the corresponding representation $U_{0}$ of $\hat{T}$ by a suitable multiplicative character $e^{i p x}$ (notice that this multiplication has the effect of translating the spectrum of $U_{0}$ by the vector $p \in \hat{T})$. The spectrum of $\overline{\mathfrak{A}}_{1}^{T}\left(\bar{V}_{+}\right)$is thereby parametrized by couples $(\pi, p)$ where $\pi$ runs through $\hat{\mathfrak{A}}_{p}$ and $p$ through a subset $S_{\pi}$ of $\hat{T}$ which a priori depends upon $\pi$. We now show that, by a suitable choice of $U_{0}$ in each " $\pi$-class", $S_{\pi}$ can be assumed to be the closed forward light cone so that the spectrum of $\overline{\mathfrak{A}}_{1}^{T}\left(\bar{V}_{+}\right)$can be identified set theoretically with the direct product $\hat{\mathfrak{A}}_{p} \times \bar{V}_{+}$. Let $\tilde{\pi}=(\pi, U)$ be an element of $\widehat{\mathfrak{A}_{1}^{T}\left(\vec{V}_{+}\right)}$: the spectrum $\Sigma_{U}$ of $U$ has the property that the smallest closed convex cone with vertex in the origin containing it is the forward closed light cone ([11] Lemma 9). Then there exists a maximal vector $p \in \bar{V}+$ such that $-p+\Sigma_{U} \subset \bar{V}_{+}$(maximal for the order relation $p_{1} \leqq p_{2} \Leftrightarrow p_{1}-p_{2} \in \bar{V}_{+}$).

Taking $U_{0}=e^{-i p x} U$ we then obtain the reference element mentioned above in our $\pi$-class (it can be characterized geometrically by the fact that its spectrum $\Sigma_{U_{0}}$ is tangent - we expect this to be at infinity - to the light cone).

The " $\bar{V}_{+}$ambiguity" in the passage from $\pi$ to $\tilde{\pi}$ explains the two following facts 
1) If $\mathfrak{A}$ is the quasi-local algebra of the free scalar neutral field with non vanishing lowest mass, $\hat{\mathfrak{A}}_{p}$ contains only one element. Nevertheless $\mathfrak{A}_{1}^{T}\left(\bar{V}_{+}\right)$is not elementary ${ }^{5}$, the $\bar{V}_{+}$ambiguity giving it $\bar{V}_{+}$as a spectrum.

2) If $\mathfrak{A}$ has an identity as in the case of free fermions $\mathfrak{A}_{1}^{T}$ includes the $L_{1}$ group algebra of the translation group $T$ so that each character $e^{i p x}$ with $p \in \bar{V}_{+}$can be extended* to an irreducible representation $(\pi, U)$ of $\mathfrak{A}_{1}^{T}(V)$ : for this representation we have a vector $\Omega$ such that

$$
U(x) \Omega=e^{i p x} \Omega, \quad x \in T .
$$

But $\Omega$ is nothing but a vacuum state for $U^{\prime}(x)=e^{-i p x} U(x)$.

Let us add a few remarks on the spectrum of $\mathfrak{A}_{1}^{T}\left(\bar{V}_{+}\right)$. It is not to be expected that its Jacobson topology is just the product topology of the relative Jacobson topology of $\hat{\mathfrak{A}}_{p}$ and the Euclidian topology of $\bar{V}_{+}$. If this were the case the Jacobson topology of $\widehat{\mathfrak{A}}_{1}^{T}\left(\bar{V}_{+}\right)$would be Hausdorff which, by definition, would mean that each primitive ideal should be maximal. We do not expect this because one intuitively thinks that inclusion of ideals is in some way connected with inclusion of spectra and, for each element $(\pi, p)$ of $\widehat{\mathfrak{A}_{1}^{T}}\left(\bar{V}_{+}\right)$, the corresponding spectrum of momenta includes the spectra corresponding to a $\left(\pi, p^{\prime}\right)$ with a suitably chosen $p^{\prime}$.

In fact the Hausdorff character of the Jacobson topology of $\widehat{\mathfrak{A}_{1}^{T}\left(\bar{V}_{+}\right)}$ would have the very strong and unlikely implication that the algebra $\overline{\mathfrak{A}}_{1}^{T}\left(\bar{V}_{+}\right)$should be liminar (see [6] $\S 3$ and 4 ) so that each irreducible representation would map it into the compact operators of Hilbert space. This we know not to be the case when $\mathfrak{A}$ has an identity (e.g. $C^{*}$-algebra generated by the field operator of the free fermion field) because in that case the $L_{1}$-algebra of the translation group $T$ is included in $\mathfrak{A}_{1}^{T}$, its representations being known not to be compact operators.

\section{\$ VI. Asymptotically Abelian algebras}

We now wish to consider the formalism developed in section II-IV in the context of certain special algebras which we call asymptotically Abelian and which we define as follows.

Definition 4. Let $\mathfrak{A}$ be a $C^{*}$-algebra acted upon by an Abelian group $T$ of automorphisms with the properties i) to iii) of section II. We call $\mathfrak{A}$ asymptotically Abelian if the following condition is satisfied for all $A, B \in \mathfrak{A}$

$$
\|[A(\mathbf{x}), B]\| \underset{\mathbf{x} \rightarrow \infty}{\longrightarrow} 0 .
$$

5 See [6], 4.7.3.

* If $\mathfrak{A}_{1}^{T}(V)$ is completely symmetric cf. note added in proof below. 
This definition is naturally motivated by physical considerations and covers the quasi-local algebras of both relativistic field theory and statistical mechanics. In contrast to the situation in the previous section $T$ will now be just the group of space translations $\mathrm{x}$ and in the following $\mathfrak{A}_{1}^{T}$ is the covariance algebra constructed with these three-dimensional translations.

We are now going to restrict our attention to a special class of states $\Phi$ over $\mathfrak{A}$ and analyse the consequences of the asymptotic Abelian structure. We recall

Definition 5. A state (i.e. a positive linear form) $\Phi$ over $\mathfrak{A}$ is called invariant if

for all $A \in \mathfrak{A}$ and $\mathbf{x} \in T$.

$$
\Phi(A(\mathrm{x}))=\Phi(A)
$$

Definition 6. $A$ state $\Phi$ over $\mathfrak{A}$ is said to be clustering if, for all $A, B \in \mathfrak{A}$

$$
\Phi(A(\mathrm{x}) B)-\Phi(A(\mathrm{x})) \Phi(B) \underset{|\mathbf{x}| \rightarrow \infty}{\longrightarrow} 0 .
$$

Now however we want to use a new definition whose role will appear in the sequel

Definition 7. We first introduce the following notation. We write for a function $g$ over $R_{3}$

$$
M(g(.))=\lim \frac{1}{\frac{4 \pi}{3} L^{3}} \int_{|\mathbf{x}| \leqq L} g(\mathbf{x}) d \mathbf{x}
$$

whenever the limit exists. $M(g)$ is called the Wiener mean of $g$. Now a state will be called weakly clustering whenever the Wiener mean of the expression (50) vanishes.

We will need the following properties of the mean (51):

A) If $g(x)$ is continuous and vanishes at infinity i.e.

$$
g(\mathbf{x}) \underset{|\mathbf{x}| \rightarrow \infty}{\longrightarrow} 0
$$

then $M(g())=$.0 (consequently every clustering state is weakly clustering).

B) Take $g$ to be the Fourier transform of a bounded measure $\mu$. Then

$$
M\left(|g(.)|^{2}\right)=\sum_{p \in R_{3}}|\mu(\{p\})|^{2} .
$$

In the last sum only the points $p_{n}$ contribute which have a discrete weight for the measure $\mu$, in other terms this sum refers to the discrete part of the measure $\mu$.

C) If $g$ is the Fourier transform of a continuous bounded measure (i.e. one without discrete masses) then $M(g())=$.0 . 
A) is an immediate consequence of the definition (51). B) can be adapted from a well known theorem of Wiener on Fourier series ([12] vol. I p. 108, [13] p. 118)

$$
|M(g(.))|^{2} \leqq M\left(|g(.)|^{2}\right)
$$

which is in turn a straightforward consequence of the Schwartz inequality.

D) If $g$ is the Fourier transform of a bounded measure $\mu$ then

$$
M(g(.))=\mu(\{0\}),
$$

i.e. the mean value of $g$ is the value of $\mu$ at the origin which is of course non-zero if $\mu$ has a discrete part at the origin. Statement $\mathrm{D}$ may be deduced from $C$.

In the following we will consider, in addition to invariant states $\Phi$ over $\mathfrak{A}$, the states $\hat{\Phi}$ over $\mathfrak{A}_{1}^{T}$ constructed from the $\Phi$ in the following manner

$$
\hat{\Phi}(X)=\int \Phi\left(X_{\mathbf{u}}\right) d \mathbf{u} \quad X \in \mathfrak{R}_{1}^{T}
$$

the passage from $\hat{\Phi}$ to $\Phi$ is the inverse of the process $\hat{\Phi} \rightarrow \Phi$ described by Theorem 4 of section 2 (unique in the case of an invariant state). We notice that $\hat{\Phi}$ is invariant as well as $\Phi$ :

$$
\hat{\Phi}(X(\mathrm{x}))=\hat{\Phi}(X) \quad X \in \mathfrak{R}_{1}^{T}, x \in T
$$

where $X(\mathrm{x})$ is the translate of $X$ by $\mathrm{x}$ defined by $\{X(\mathrm{x})\}_{u}=X_{u}(\mathrm{x})$, $u \in T$.

Given an invariant state $\Phi$ on $\mathfrak{A}$ it is well known that the GelfandSegal construction [14] delivers a covariant representation $(\pi, U)$ of $\mathfrak{A}$ acting in a Hilbert space $\mathscr{H}$ in such a way that

and

$$
\Phi(A)=(\Omega|\pi(A)| \Omega)
$$

$$
U(\mathrm{x}) \Omega=\Omega,
$$

where $\Omega \in \mathscr{H}$ is cyclic for $\pi(\mathfrak{Q})$. The Gelfand-Segal construction applied to the state (54) on $\mathfrak{A}_{1}^{T}$ delivers a representation $\hat{\pi}$ of $\mathfrak{A}_{1}^{T}$ acting in the same Hilbert space $\mathscr{H}$ such that, in conformity with Theorems 3 and 4

and

$$
\Phi(X)=(\Omega|\pi(X)| \Omega) \quad X \in \mathfrak{A}_{1}^{T}
$$

$$
\pi(X)=\int \pi\left(X_{\mathbf{x}}\right) U(\mathbf{x}) d \mathbf{x} \quad X \in \mathfrak{A}_{1}^{T},
$$

$\Omega \in \mathscr{H}$ being also cyclic for $\hat{\pi}\left(\mathfrak{A}_{1}^{T}\right)$.

We will now derive structure properties of the invariant states $\Phi$ and their associated representations, whereby we will use the notations introduced in the last paragraph.

${ }^{6}$ We are indebted to Prof. P. Billard for pointing out this fact to us. 
Let us first consider the spectrum of $U(x)$. A priori it contains both a discrete and a continuous spectrum and we write accordingly

$$
U(\mathbf{x})=\sum_{p_{n}} E\left(p_{n}\right) e^{i \mathbf{p}_{n} \cdot \mathbf{x}}+\int e^{i \mathbf{p} \cdot \mathbf{x}} d E(\mathbf{p})
$$

where $d(\psi|E(\mathbf{p})| \varphi)$ is a continuous measure for all $\psi, \varphi \in \mathscr{H}$ and $E\left(\mathbf{p}_{n}\right)$ is the (possibly infinite dimensional) projector onto all states $|n\rangle$ in $\mathscr{H}$ such that

$$
U(\mathbf{x})|n\rangle=e^{i \mathbf{p}_{n} \cdot \mathbf{x}}|n\rangle .
$$

Of course one has $E(0) \Omega=\Omega$. We now prove the

Lemma 6. For an asymptotically Abelian algebra $\mathfrak{A}$ we have

and

$$
\left[E\left(p_{n}\right) \pi(A) E\left(p_{n}\right), E\left(p_{n}\right) \pi(B) E\left(p_{n}\right)\right]=0 \quad A, B \in \mathfrak{A}
$$

$$
M\{(\varphi|U(.)-E(0)| \psi)\}=0 .
$$

Proof: Let us define, for arbitrary $\psi, \varphi \in \mathscr{H}$

$$
\begin{aligned}
g_{n}(x)= & \left(\psi\left|E\left(\mathbf{p}_{n}\right)[\pi(A(\mathbf{x})), \pi(B)]\right| E\left(\mathbf{p}_{n}\right) \mid \varphi\right) \\
= & \left(\psi\left|E\left(\mathbf{p}_{n}\right) \pi(A) U(-\mathbf{x}) \pi(B) E\left(\mathbf{p}_{n}\right)\right| \varphi\right) e^{i \mathbf{p}_{n} \cdot \mathbf{x}}- \\
& -\left(\psi\left|E\left(\mathbf{p}_{n}\right) \pi(B) U(\mathbf{x}) \pi(A) E\left(\mathbf{p}_{n}\right)\right| \varphi\right) e^{i \mathbf{p}_{n} \cdot \mathbf{x}} .
\end{aligned}
$$

Due to the asymptotic Abelian character

$$
g_{n}(x) \underset{|\mathbf{x}| \rightarrow \infty}{\longrightarrow} 0,
$$

from which we conclude that

$$
M\left\{\left|g_{n}(.)\right|^{2}\right\}=0 .
$$

Now $g_{n}$ is the Fourier transform of a measure so that we may (52) use to conclude that

$E\left(\mathbf{p}_{n}\right) \pi(A) E\left(\mathbf{p}_{n}-p_{m}\right) \pi(B) E\left(\mathbf{p}_{n}\right)=E\left(\mathbf{p}_{n}\right) \pi(B) E\left(\mathbf{p}_{m}+\mathbf{p}_{n}\right) \pi(A) E\left(\mathbf{p}_{n}\right)$.

In particular for $\mathbf{p}_{m}=0$ we see that part I of the Lemma is established. The second part of the Lemma follows directly from property D) quoted above.

We have now considered the case where we have invariant states, but there remains the possibility that $E(0)$ is more than one-dimensional. However in the case that it is one-dimensional we have the remarkable structure stated in the

Theorem 7. If $\Phi$ is an invariant state over an asymptotically Abelian algebra $\mathfrak{A}, \Phi$ the invariant extension of $\Phi$ to $\mathfrak{A}_{1}^{T}$ and $\pi$ and $\hat{\pi}$ the corresponding representations of these algebras on a Hilbert space $\mathscr{H}$ with cyclic vector $\Omega$ determined by $\Phi$ and $\hat{\Phi}$ we have equivalence of the following statements

1) Weak clustering of $\Phi$ :

$$
M\{\Phi(A(.) B)\}=\Phi(A) \Phi(B) .
$$


2) For all $\varphi, \psi \in \mathscr{H}$

$$
M\{(\psi|U(.)| \varphi)\}=\left(\psi\left|E_{\Omega}\right| \varphi\right),
$$

where $E_{\Omega}$ denotes the projector on the vector $\Omega$.

3) For all $\varphi, \psi \in \mathscr{H}$ and $A \in \mathfrak{A}_{1}^{T}$

$$
M\{(\psi|\pi(A(.))| \varphi)\}=(\Omega|\pi(A)| \Omega)(\psi \mid \varphi) .
$$

4) $\hat{\Phi}$ is a pure state of $\mathfrak{A}_{1}^{T}$ i.e. $\hat{\pi}\left(\mathfrak{Q}_{1}^{T}\right)$ is irreducible.

5) $\Omega$ is the only invariant vector of $\mathscr{H}$ i.e.

$$
E(0)=E_{\Omega} .
$$

Proof: 1) and 2) are two ways of writing the same fact owing to the cyclicity of $\Omega$ for $\pi(\mathfrak{A})$. The equivalence of 2) and 3 ) is supplied by the straightforward identity

$$
\begin{aligned}
& (\Omega|\pi(B)[\pi(A(\mathrm{x}))-(\Omega \mid \pi(A) \Omega)] \pi(C)| \Omega) \\
& \quad=(\Omega|\pi(B)[\pi(A(\mathrm{x})), \pi(C)]| \Omega)+\left(\Omega\left|\pi(B) \pi(C)\left[U(\mathrm{x})-E_{\Omega}\right] \pi(A)\right| \Omega\right)
\end{aligned}
$$

upon taking mean values (the first term on the right hand side has mean value zero because of property A) above).

Let us now show that 2) implies 4). Take $C \in \pi\left(\mathfrak{A}_{1}^{T}\right)^{\prime}$. Then $C$ commutes with all $U(\mathrm{x})$ (see end of Theorem 3). Now, for any $X, Y \in \mathfrak{A}_{1}^{T}$

$$
\begin{aligned}
(\Omega|\hat{\pi}(X)[C-(\Omega|C| \Omega)] \hat{\pi}(Y)| \Omega) & =(\Omega|\hat{\pi}(X) \hat{\pi}(Y)[C-(\Omega|C| \Omega)]| \Omega) \\
& =M\left\{\left(\Omega\left|\hat{\pi}(X) \hat{\pi}(Y)\left[U(.)-E_{\Omega}\right] C\right| \Omega\right)\right\} \\
& =0
\end{aligned}
$$

i.e. $C$ is a scalar and $\hat{\pi}$ is irreducible.

Next we demonstrate that 5) follows from 4). We first note that

$$
\hat{\pi}(X) E(0)=\pi\left\{\int X_{\mathbf{u}} d \mathrm{u}\right\} E(0)
$$

and therefore deduce from Lemma 6 that $E(0) \hat{\pi}\left(\mathfrak{A}_{1}^{T}\right) E(0)$ is Abelian. This property is incompatible with the irreducibility of $\hat{\pi}$ unless $E(0)$ is one-dimensional i.e. $E(0)=E_{\Omega}$.

Now, using this fact and (61) of Lemma 6, we see that 2) is a direct consequence of 5) which completes the proof of the theorem.

It is seen from the above theorem that the invariant states described therein have physical characteristics which commend them as candidates for equilibrium states in statistical mechanics. We would finally like to demonstrate that if we have two asymptotically Abelian algebras $\mathfrak{A}$ and $\mathfrak{B}$ such that $\mathfrak{A} \subset \mathfrak{B}$ then it is possible to extend an invariant weakly clustering state over $\mathfrak{A}$ to a state with similar properties over $\mathfrak{B}$.

Theorem 8. If $\mathfrak{A}$ and $\mathfrak{B}$ are asymptotically Abelian algebras such that $\mathfrak{A} \subset \mathfrak{B}$ and $\Phi_{\mathfrak{A}}$ is an invariant weakly clustering state over $\mathfrak{A}$ then there exists at least one invariant weakly clustering state $\Phi_{\mathfrak{B}}$ over $\mathfrak{B}$ such that

$$
\Phi_{\mathfrak{A}}(A)=\Phi_{\mathfrak{B}}(A) \text { for all } A \in \mathfrak{A}
$$


Proof. Let us first form the two covariant algebras $\mathfrak{2}_{1}^{T}$ and $\mathfrak{B}_{1}^{T}$ and note that as $\mathfrak{A} \subset \mathfrak{B}, \mathfrak{A}_{1}^{T} \subset \mathfrak{B}_{1}^{T}$ by construction. Now by (54) we can construct from $\Phi_{\mathfrak{Q}}$ an invariant state $\widehat{\Phi}_{\mathfrak{A}}$ over $\mathfrak{A}_{1}^{T}$ and it follows from Theorem 7 that $\hat{\Phi}_{\mathfrak{A}}$ is pure. It is known that a pure state over a subalgebra can always be extended to a pure state over the full algebra; hence we can extend $\hat{\Phi}_{\mathfrak{A}}$ over $\mathfrak{A}_{1}^{T}$ to a pure state $\hat{\Phi}_{\mathfrak{B}}$ over $\mathfrak{B}_{1}^{T}$. However this latter extension can be made in such a manner that $\Phi_{\mathfrak{B}}$ is an invariant state because the invariance of a state over a covariance algebra is a property of the restriction of the state to the subalgebra $L_{1}(T)$ of the translation group and as $L_{1}(T) \subset \mathfrak{A}_{1}^{T} \subset \mathfrak{B}_{1}^{T}$ then $\hat{\Phi}_{\mathfrak{A}}$ and $\hat{\Phi}_{\mathfrak{B}}$ coincide over $L_{1}(T)$ (if $\mathcal{A}$ does not contain anidentity then $L_{1}(T)$ is not strictly a subalgebra of $\mathfrak{X}_{1}^{T}$ but the preceding argument is nevertheless possible with the use of an approximate identity). Finally we note that as $\hat{\Phi}_{\mathfrak{B}}$ is an invariant pure state over $\mathfrak{B}_{1}^{T}$ then the state $\Phi_{\mathfrak{B}}$ over $\mathfrak{B}$ derived from $\hat{\Phi}_{\mathfrak{Z}}$ (cf. Theorem 4) is a weakly clustering state by Theorem 7 .

We note that the concept of covariance algebras is essential for deriving Theorem 8 . This theorem solves to a considerable extent a problem recently raised by one of us [17] in the context of the canonical commutation relations. In this latter context one must consider an algebra $\mathfrak{A}$ of physically observable currents, heuristically described by expressions such as $\varphi^{+}(x) \varphi(y)$ where $\varphi^{+}(x), \varphi(y)$ are creation and annihilation operators, but it is mathematically more convenient to consider a larger algebra $\mathfrak{B}$ which, again in a heuristic sense, contains the non-observable fields $\varphi(x)$ and $\varphi^{+}(y)$. Theorem 8 tells us that if we consider only invariant weakly clustering states then we can, without loss of generality, work with either algebra. Although the theorem has no direct relevance to the extension from observables to fields when the fields satisfy anti-commutation relations it is however straightforward to develop a similar result in this case.

We conclude by commenting on the equivalences given by Theorem 7 . The equivalence of properties 3) and 4) is a generalisation of a Lemma due to HAAG $_{\text {[15] }}$ and the equivalence of 1), 4) and 5) is a generalisation of the well known equivalence of the uniqueness of $\Omega$, irreducibility and clustering. However in our general framework irreducibility is of $\mathfrak{A}_{1}^{T}$, and not of $\mathfrak{A}$, as would be the case in relativistic field theory with the added assumption of positive energy, and the clustering property which is relevant is the weak and not the strong clustering. These two differences can be understood if one comments that the positive energy assumption firstly ensures that $U(x)$ is in the weak closure of $\pi(\mathfrak{A})$ [9] and secondly places strong continuity properties on the spectral measure associated with $U(x)$ [16]. In statistical mechanics the weak clustering property is probably most appropriate for characterising the absence of long range correlations at large distances even in systems with a periodic structure. 


\section{Appendix}

In the following we wish to comment upon the situation where $\mathfrak{A}$ is known to have a faithful representation in which $T$ is unitarily implemented without assuming separability of $\mathfrak{A}$. In this case we cannot derive assumption iii) of $\S I I$, however we will show that $\mathfrak{A}$ can be replaced by a "regularized" $C^{*}$-algebra $\underline{I}$ whose set of covariant representations includes those of $\mathfrak{A}$.

Let us call $\mathscr{E}_{0}$ the subset of the state space consisting of all states $\varphi$ such that $x \rightarrow \varphi\left(B^{*} A(x) B\right)$ is a measurable function for all $A, B \in \mathfrak{A}$. $\mathscr{E}_{0}$ is non-void (it is even weakly dense in $\mathfrak{A}^{*}$ ), norm-closed, invariant in translations by elements of the algebra and invariant in the group $T$. Consequently the linear hull $\mathscr{X}$ is the norm-closed set of ultraweakly continuous forms in some representation $\tau$ ( $\tau$ is the sum of all representations constructed with states in $\mathscr{E}_{0}$ ) and the dual space $\mathfrak{X}^{*}$ of $\mathscr{X}$ can be identified with the von Neumann algebra generated by $\tau(\mathfrak{Q})$ so that $\mathfrak{A}$ can be considered as a $C^{*}$-subalgebra of $\mathfrak{X}^{*}$.

Given $A \in \mathfrak{A}$ and $\chi \in L_{1}(T)$ we then see that we define a $A_{\chi} \in \mathfrak{X}^{*}$ by defining its value for an arbitrary $\varphi \in \mathcal{X}$ as

$$
\varphi\left(A_{\chi}\right)=\int \chi(x) \varphi(A(x)) d x
$$

whereby

$$
\left\|A_{\chi}\right\| \leqq\|A\| \cdot\|\chi\|_{1} .
$$

Our $\mathfrak{A}$ is then defined as the $C^{*}$-subalgebra of $\mathfrak{X}^{*}$ generated by all $A_{\chi}$, $A \in \hat{\mathcal{A}}, \chi \in L_{1}(T)$. The action of $T$ extends to $\mathfrak{X}^{*}$ by double transposition. Furthermore since

$$
A_{\chi}(x)=A_{\chi}(\cdot-x)
$$

for all $A \in \mathfrak{A}, x \in T$ and $\chi \in L_{1}(T)$ we see that $T$ leaves invariant the set of $A_{\chi}$ and is consequently represented in a strongly continuous way (see (62)) in the automorphism group of $\mathfrak{A}$.

Let now $(\pi, U)$ be a covariant representation of $\mathfrak{A}, \pi$ is then quasiequivalent to a subrepresentation of $\tau$ and therefore extends to a $W^{*}$. representation of $\mathfrak{H}^{*}=\tau(\mathfrak{A})^{\prime \prime}$ whose restriction to $\mathfrak{H}$ affords a covariant representation $\left(\underline{\pi}, U\right.$ ) of this algebra such that $\underline{\pi}(\underline{\mathfrak{Q}})^{\prime \prime}=\pi(\mathfrak{A})^{\prime \prime}$ (this is seen using the theorem of the bipolar). In the case where $\mathfrak{A}$ has a local structure $O \rightarrow \mathfrak{A}(O)$ we can analogously define a local structure $O \rightarrow \underline{\mathfrak{A}}(O)$ of $\underline{\mathfrak{A}}$ such that $\underline{\mathfrak{x}}(\underline{\mathfrak{A}}(O))^{\prime \prime}=\pi(\mathfrak{A}(O))^{\prime \prime}$.

We now prove injectivity of the mapping $\pi \rightarrow \underline{\pi}$ by showing how to recover $\pi$ from $\underline{\pi}$. Generally, given a representation $\underline{\pi}$ of $\mathfrak{A}$ and a $A \in \mathfrak{A}$ let us consider the set of operators $\underline{\pi}\left(A_{z_{\alpha}}\right)$ where $\chi_{\alpha}$ is an approximate unit in $L_{1}(T)$. This set is, by (62), contained in the ball of radius $\|A\|$ in the set of bounded operators on the representation space. Since this ball is compact for the weak topology of operators on the representation 
space there is a finer filter $\left\{\alpha^{\prime}\right\}$ for which we have weak convergence of the operators $\underline{\pi}\left(A_{\chi_{\alpha}}\right)$ towards an operator $\sigma_{\left\{\alpha^{\prime}\right\}}(A)$ which we now show to coincide with $\pi(A)$. For this let us notice that the definition

$$
B_{\eta}=\int \eta(x) U(x) B U(x)^{-1} d x
$$

where $\eta$ is continuous with compact support, determines a weakly continuous map $B \rightarrow B_{\eta}$ on the bounded operators of the representation space. We then have that

$$
\text { weak } \underset{\alpha^{\prime}}{\operatorname{limit}} \underline{\pi}\left(A_{\chi_{\alpha^{\prime}} * \eta}\right)=\left[\sigma_{\left\{\alpha^{\prime}\right\}}(A)\right]_{\eta}=\underline{\pi}\left(A_{\eta}\right)=[\pi(A)]_{\eta}
$$

from which the conclusion follows due to the arbitrariness of $\eta$.

We notice that the same procedure applied to an arbitrary covariant representation $\pi^{\prime}$ of $\underline{\mathfrak{U}}$ instead of $\underline{\pi}$ yields a correspondence $A \rightarrow \sigma(A)$ which is easily seen to be linear, continuous and positive. We leave open the question of whether $\sigma$ is a representation of $\mathfrak{A}$ - which would imply that the map $\pi \rightarrow \underline{\pi}$ is bijective. We limit ourselves to noticing that a proof of denumerability of the superselection sectors for II would imply the same fact for $\mathfrak{A}$.

Acknowledgments. The authors are happy to acknowledge a number of useful and instructive discussions with Prof. J. GLIMm whose comments were very helpful especially in connection with Section V. Useful discussions were also held with Profs. P. Billaard, N. Hugenholtz and D. Ruelle in connection with Section VI. We thank D. Ruelle for providing us with a copy of a work to be published in which he develops independently some of the notions presented in Section VI of this paper. The authors are indebted to the Ministère de l'Education Nationale Paris - for granting two of them (S. Doplicher and D. W. Robinson) exchange professorships which made their collaboration possible.

Note added in proof. The proof of theorem 8 in $\S$ VI is insufficient as was pointed out by D. RUELLE who provided a counter example. However the proof would be valid if the covariance algebras $\mathfrak{Q}_{1}^{T}$ on $\mathscr{L}_{1}^{T}$ where completely symmetric Banach *-algebras. We conjecture that this is the case for a covariance algebra $\mathfrak{U}_{1}^{T}$ whenever the group $T$ posseses invariant means (in particular, if $T$ is abelian).

\section{References}

[1] HaAG, R., and D. Kastler: J. Math. Phys. 5, 848 (1964).

[2] Hille, E., and R. S. Phillips: Functional analysis and semi-groups; Am. Math. Soc. Coll. Pub. Providence R.I. (1957).

[3] Bourbaki, N.: Eléments de mathématiques, Livre VI: Intégration. Paris: Hermann 1952.

[4] Dixmier, J.: Comment. Math. Helv. 26, 275 (1952).

[5] GLrmm, J.: Pacific J. Math. 12, 885 (1962).

[6] Drxmrer, J.: Les $C^{*}$-algèbres et leurs représentations. Paris: GauthierVillars 1964.

[7] Varopoulos, N. Th.: C.R. Acad. Sci. 258, 2465 (1964). 
[8] Neumark, M. A.: Normierte Algebren, Berlin: VEB Deutscher Verlag der Wissenschaften 1959.

[9] Borchers, H. J.: Energy and momentum as observables. Preprint.

[10] - Lecture notes, Cargèse Summer School (1965).

[11] - Commun. Math. Phys. 1, 49 (1965).

[12] Zxgmund, A.: Trigonometric Series 2nd ed. vol. I. Cambridge: Univ. Press 1959.

[13] Rudin, W.: Fourier analysis on groups. New York: Interscience Publ. 1962.

[14] Segal, I. E.: Bull. Am. Math. Soc. 53, 73 (1947).

[15] HAAG, R.: Nuovo cimento 25, 287 (1962).

[16] Borchers, H. J.: Local rings and the connection of spin with statistics. Preprint.

[17] Robinson, D. W.: Commun. math. Phys. 1, 159 (1965).

[18] Turumaru, T.: Tohoku Math. Journal 10, 355 (1958). 